\title{
Differences in RNA polymerase II complexes and their interactions with surrounding chromatin on human and cytomegalovirus genomes
}

\section{Benjamin Spector}

Carver College of Medicine University of lowa

\section{Mrutyunjaya Parida}

Carver College of Medicine University of lowa

Christopher Ball

Carver College of Medicine University of lowa

Ming Li

Carver College of Medicine University of lowa https://orcid.org/0000-0003-0396-4078

\section{Jeffrey Meier}

Carver College of Medicine University of lowa

Donal S. Luse

Lerner Research Institute

David Price ( $\boldsymbol{\sigma}$ david-price@uiowa.edu )

Carver College of Medicine University of lowa

\section{Genetics Article}

Keywords: RNA polymerase II, preinitiation complexes, chromatin, human cytomegalovirus, TBP, UL87, DFF ChIP-Seq

Posted Date: October 8th, 2021

DOI: https://doi.org/10.21203/rs.3.rs-912323/v1

License: (a) (i) This work is licensed under a Creative Commons Attribution 4.0 International License. Read Full License

Version of Record: A version of this preprint was published at Nature Communications on April 14th, 2022. See the published version at https://doi.org/10.1038/s41467-022-29739-x. 


\section{Differences in RNA polymerase II complexes and their interactions with surrounding chromatin on human and cytomegalovirus genomes}

Benjamin M. Spector ${ }^{a}$, Mrutyunjaya Paridaa, Ming Li $i^{a, b, c, d}$, Christopher B. Balla, Jeffrey L. Meier $^{b, c, d}$, Donal S. Luse ${ }^{e}$, and David H. Price ${ }^{a}$

aDepartment of Biochemistry, The University of lowa, lowa City, IA 52242, USA

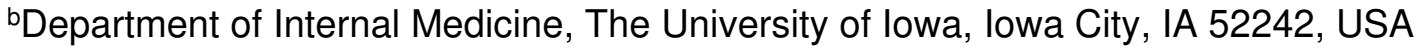

'Department of Epidemiology, The University of lowa, lowa City, IA 52242, USA

dVeterans Affairs Health Care System, lowa City, IA 52242, USA

eDepartment of Cardiovascular and Metabolic Sciences, Lerner Research Institute, Cleveland Clinic, Cleveland, OH, 44195, USA.

Keywords: RNA polymerase II, preinitiation complexes, chromatin, human cytomegalovirus, TBP, UL87, DFF ChIP-Seq

\section{Summary}

Interactions of the RNA polymerase II (PoI II) preinitiation complex (PIC) and paused early elongation complexes with the first downstream (+1) nucleosome are thought to be functionally important. However, current methods are limited for investigating these relationships, both for cellular chromatin and the human cytomegalovirus (HCMV) genome. Digestion with human DNA fragmentation factor (DFF) before immunoprecipitation (DFF-ChIP) precisely revealed both similarities and major differences in PICs driven by TBP on the host genome in comparison with PICs driven by TBP or the viral-specific, late initiation factor UL87 on the viral genome. Host PICs and paused Pol II complexes are frequently found in contact with the +1 nucleosome and paused Pol II can also be found in a complex involved in the initial invasion of the +1 nucleosome. In contrast, viral transcription complexes have very limited nucleosomal interactions, reflecting a relative lack of chromatinization of transcriptionally active regions of HCMV genomes.

\section{Highlights}

DFF-ChIP and fragMaps allow visualization of large transcription complexes in cells HCMV promoters are not surrounded by H3K4me3 marked nucleosomes

PICs are major features found on host and viral genomes

PICs driven by TBP and the HCMV late transcription factor UL87 are highly dissimilar 


\section{Introduction}

Regulation of human gene expression is accomplished by a highly orchestrated interplay between the transcription machinery and its chromatinized genomic template. The required general Pol II initiation factors are instructed by Mediator and a host of more specific transcription factors to utilize selected promoters as sites of initiation ${ }^{1}$. However, the default state of the genome is repressive because of global nucleosome deposition, which must be relieved by chromatin remodelers. Assembly of the preinitiation complex (PIC) occurs over sequences surrounding the TSS and an upstream region depleted of nucleosomes (NDR $)^{2}$. A strongly positioned +1 nucleosome has a boundary around $50 \mathrm{bp}$ downstream from the transcription start site (TSS) ${ }^{3}$. This nucleosome and several downstream nucleosomes are marked by tri-methylation of histone $\mathrm{H} 3$ on lysine $4(\mathrm{H} 3 \mathrm{~K} 4 \mathrm{me} 3)^{4,5}$. Both PIC assembly and pausing by newly-initiated Pol II have been linked to interactions with the +1 nucleosome ${ }^{6-10}$, but the nature and functional significance of these interactions remain incompletely understood. Existing global methods to visualize and quantify sites of transcription and the locations of the transcriptional machinery within the local chromatin landscape have had limited success in addressing these questions. Occupancy by the +1 nucleosome is near $100 \%$ while Pol II occupancy is generally much less than $10 \%$, resulting in a potentially misleading correlation between two disparate signals. Recently there has been a number of highly informative structural studies of PICs ${ }^{11-14}$, but the abundance of PICs on cellular chromatin has not been adequately determined and their positioning over promoter elements has been primarily inferred from in vitro studies.

The interface of transcription and chromatin on the HCMV genome is even more poorly defined. Lytic infection can propagate only in non-dividing cells. The process begins with delivery of a nucleosome-free viral genome to the nucleus, where the standard host Pol II machinery drives transcription from the major immediate early promoter. Expression of viral immediate early proteins then allow expression of early genes ${ }^{15,16}$. The early genes encode the machinery for viral DNA replication and for transcription of a group of late genes, which requires a special set of viral-specific Pol II initiation factors ${ }^{17-20}$ that likely replace some of the host initiation factors. While ChIP-PCR on individual loci and genome-wide studies have revealed changes in chromatinization throughout the viral lifecycle ${ }^{21-24}$, it is not clear what regions on each of the hundreds of viral genomes present late in infection are occupied by nucleosomes. Nothing is known about how Pol II transcription complexes interface with any chromatin that may be present. Our recent work demonstrated promiscuous transcription initiation from thousands of promoters across the $\sim 240,000$ bp dsDNA genome, consistent with the idea that chromatinization of the HCMV genomes during lytic infection is incomplete ${ }^{25}$. Increasing our knowledge of how HCMV transcription is regulated is important for identification of potential therapeutic targets since the virus infects about $60 \%$ of the population. HCMV is a significant cause of death in immunocompromised individuals ${ }^{26}$ and a leading viral cause of birth defects ${ }^{27}$.

Our group recently described a nuclear run-off method to directly observe engaged Pol II interacting with the +1 nucleosome ${ }^{6}$. We digested nuclei with the double-stranded endonuclease human DNA fragmentation factor (DFF) and then chased nascent transcripts to the resulting run-off sites. We found that many, but not all, paused polymerases were abutted to the +1 nucleosome. Because DFF digestion preserved the viability of transcription complexes and their relation to chromatin, we decided to investigate whether combining it with chromatin immunoprecipitation would allow improved insight into localization and positioning of transcription complexes. We found that we were able to quantitatively visualize PICs as well as interactions between PICs and paused transcription complexes with the downstream chromatin. Most of our experiments were done on primary human foreskin fibroblasts (HFFs) productively infected with HCMV, allowing a direct comparison of the chromatin neighborhoods of human genomic Pol II promoters with their more poorly characterized counterparts on the viral genome. 


\section{Results}

Our study began with a characterization of our initial DFF-Seq dataset (GSE139237) generated from HeLa cells ${ }^{6}$. Fragments from DFF-Seq were about $160 \mathrm{bp}$ in length and primarily derived from nucleosomes covering inactive regions of the genome, but a very small percentage of fragments were sub-nucleosomal in size and many of these localized within promoter regions of actively transcribed genes ${ }^{6}$. To investigate the regions around promoters, active genes in HeLa cells were identified by truQuant ${ }^{28}$ which finds the most highly utilized TSS (MaxTSS) for each expressed gene from HeLa NasCap ${ }^{25}$ data. Fragments generated by DFF that were present in a $2000 \mathrm{bp}$ region centered on the MaxTSS of each of the 12,201 genes were collected and the distribution of fragment lengths was quantified and compared to fragment lengths from the total DFF-Seq dataset (Supplementary Fig. 1A). In the total dataset, peaks of fragment sizes corresponding to mono-, di-, and tri-nucleosome were 163, 326, and 512 bp respectively, while those values were 161, 298 and 452 in the truQuant subset. This suggests that many nucleosomes around promoters are essentially close packed. DFF is a homodimer that mostly cuts DNA to form blunt ends ${ }^{29}$. To determine any sequence requirements for DFF cleavage, sequences surrounding 520 million cut sites were examined. A slight sequence preference was seen (Supplementary Fig. 1B) with $6.8 \%$ of the sites having AAANT(cut) directly on one of the two sides of the cut. There was no preference to having this sequence on both sides.

\section{DFF-ChIP reveals differing promoter architecture on human and HCMV genomes}

Because DFF can generate primarily nucleosome-sized fragments without significant internal cutting, we performed two initial experiments (Exp1 and Exp2) to explore its use as a front end for H3K4me3 and Pol II ChIP. Nuclei from non-crosslinked HeLa or MRC5 cells expressing a GFP-tagged Pol II ${ }^{30}$ were digested with DFF for 1 hour to generate primarily mononucleosomes. The resulting chromatin was immunoprecipitated with antibodies to H3K4me3 modified nucleosomes or to GFP and the associated DNA was prepared for sequencing (Fig. 1A).

The DFF-ChIP results were compared to NasCap PRO-Seq data ${ }^{6}$ from HeLa cells. Paused Pol II is evident from the tall peaks in the PRO-Seq data and over a 500,000 bp region of the human genome peaks of Pol II and H3K4me3 occupancy in the DFF-ChIP tracks exhibited strong visual correlation (Fig. 1B). Greater detail can be observed across a 30,000 bp region (Fig. 1C). Each promoter exhibits clear nucleosome phasing in the H3K4me3 dataset surrounding a NDR that supports Pol II initiation in both cell types. Additionally, Pol II DFF-ChIP signal overlaps with the PRO-Seq signal but also extends further downstream. This downstream signal likely results from Pol II that is abutted to the +1 nucleosome such that DFF cannot cleave between polymerase and the nucleosome ${ }^{6}$. The complex $\mathrm{H} 3 \mathrm{~K} 4 \mathrm{me} 3$ and Pol II patterning on the majority of promoters is well replicated even across cell types and growth conditions between Exp1 and Exp2 indicating the robustness of the DFF-ChIP method. To further validate this method's reproducibility, reads found in a $10 \mathrm{~kb}$ window centered on each of 12,201 truQuant MaxTSSs from the different experiments were directly compared and strongly correlated between the datasets (Fig. 1D).

Given the success of these initial experiments, DFF-ChIP was then applied to contact inhibited HFFs infected with HCMV (TB40/E) for 48 hours (Exp3). DFF-ChIP results were compared to PRO-Seq data from similarly infected HFFs for broad regions across the host (Fig. 2A) and viral genomes (Fig. 2B). As expected on the host genome, Pol II and H3K4me3 correspond with paused Pol II evident from the PRO-Seq data. The viral genome was pervasively transcribed as previously demonstrated ${ }^{25}$ and Pol II DFF-ChIP correlated with the PRO-Seq signal when transcription of both strands is taken into account. Unlike the host genome where $\mathrm{H} 3 \mathrm{~K} 4 \mathrm{me} 3$ is found only around promoter regions, the entirety of the HCMV genome is covered with $\mathrm{H} 3 \mathrm{~K} 4 \mathrm{me} 3$, at levels ranging from relatively low to more enriched irrespective of Pol II occupancy. 
Additional insight was obtained when the results from individual promoters on the host and viral genomes were examined. The host GAPDH promoter features nucleosome phasing around a NDR that supports Pol II initiation (Fig. 2C and 2D) as it did in Exp1 and Exp2. In contrast, the viral early gene promoter for UL4 and late gene promoter for UL76 show no obvious phasing of nucleosomes (Fig. 2E and 2F). Critically, the two viral promoters are not found in NDRs. Because initiation cannot take place when the promoter is occluded by a nucleosome $^{31,32}$, the results suggest that some of the nucleosomes detected are present only on regions of viral genomes that are not transcribed. As in Exp1 and Exp2, the Pol II DFF-ChIP signal on GAPDH overlaps with the PRO-Seq signal but also extends farther upstream and downstream of the MaxTSS (Fig. 2D). Signal from the free paused Pol II predominates for the HCMV early promoter (Fig. 2E), but is not the main signal for the late promoter (Fig. 2F). Neither viral promoter shows evidence of nucleosome-abutted Pol II. The location of upstream protection seen in all three promoters suggests the presence of Pol II in preinitiation complexes and surprisingly, those complexes predominate for the viral late promoter. A fourth experiment was performed on HCMV infected HFFs using a larger set of antibodies for ChIP (Exp4). Comparison of DFF-ChIP for H3K4me3 and Pol II across specific regions on the host and viral genomes for Exp3 and Exp4 clearly demonstrates high reproducibility of the patterns of occupancy (Supplementary Fig. 2A and 2B). In addition, a number of strong correlations between Exp3 and Exp4 were found when signals around host and viral promoters were compared (Supplementary Fig. 2C and 2D).

\section{Transcription complexes and their interactions with nearby nucleosomes can be visualized using fragMaps}

Because the patterns of DFF protection around promoters varied, fragment length analysis was explored as a means to identify specific transcription complexes. The same set of MaxTSSs for all active host genes $(12,229)$ were utilized in this analysis. The distribution of DFF-ChIP fragment sizes within a thousand bp of these TSSs from the Pol II and H3K4me3 Exp4 datasets revealed several distinctive groups of fragment sizes (Fig. 3A). A majority of H3K4me3 fragments in this window center on $158 \mathrm{bp}$ in length and a smaller population were about $294 \mathrm{bp}$ in length, corresponding to mono- and di-nucleosomes. In the Pol II dataset, two abundant fragment sizes at approximately $50 \mathrm{bp}$ and $180 \mathrm{bp}$ were the most prevalent, with a smaller population of fragment sizes around $75 \mathrm{bp}$. Fragment ranges corresponding to each of the three most common Pol II populations in the 2000 bp windows were then chosen and aligned relative to TSSs (Fig. 3B). The total amount of reads in each of these ranges was normalized to emphasize the protected footprints of the less abundant $\sim 75$ bp fragments. Both the $\sim 50$ bp and $\sim 180 \mathrm{bp}$ fragments align slightly downstream of the TSS in the pause region while the $\sim 75 \mathrm{bp}$ fragments span the TSS, as would be expected for PICs. The positioning of the $\sim 50 \mathrm{bp}$ and the $\sim 180$ bp fragments are consistent with free paused Pol II and Pol II abutted to the +1 nucleosome. The same analysis was performed utilizing Exp3 data, which gave similar results (Supplementary Fig. 3A and 3B)

Since metaplots limit how many fragment lengths can be shown together in a meaningful and accurate way, we created a method that more holistically depicts the distribution of all fragments around TSSs. The output of this visualization method simultaneously captures the size, amount, and position of each fragment across all promoters in an easily viewable fragMap. For each fragment length, the coverage at each position $+/-1000 \mathrm{bp}$ around all TSSs was calculated and averaged. These averages for each fragment length at each position were then stacked with the shortest fragments on top and longest on the bottom. Fragment lengths included in this view span from 18 to $400 \mathrm{bp}$. More focused fragMaps were also created +/- 100 bp around the MaxTSS using 18 to 120 bp fragments. For most fragMaps, black values (overall darkness of the image) are set by the maximum average read value in the window. Because the black value in each fragMap is influenced by the recovery of the IP, absolute amounts of visible 
complexes should only be compared within the same fragMap. Host HFF fragMaps were generated utilizing the 12,229 TSSs found with truQuant and TSSs on the HCMV genome were found utilizing transcription start regions (TSRs) identified using TSR-finder on a PRO-Cap dataset generated from HCMV infected HFFs that resulted in 1,461 non-overlapping 200 bp regions centered on a MaxTSS ${ }^{25}$.

FragMaps for the Pol II and H3K4me3 Exp4 datasets generated a snapshot of transcription complexes and chromatin features on the host and HCMV genomes. Host H3K4me3 fragMaps show very well positioned nucleosomes relative to the TSS and a clear NDR (Fig. 3C). This patterning of nucleosomes closely resembles fragMaps generated using the DFF-Seq HeLa truQuant dataset, except that the signal becomes fainter as the distance from the TSS increases (Supplementary Fig. 3C). Pol II fragMaps of the host genome show free paused Pol II ( 50 bp fragments downstream from the TSS), Pol II positioned over the TSS ( 75 bp fragments), Pol II abutted to the +1 nucleosome ( 180 bp fragments downstream of the TSS), and Pol II associated with the first two nucleosomes ( 320 bp fragments downstream of the TSS) (Fig. 3C). The size of the fragments bearing Pol II associated with the first two nucleosomes supports the finding that nucleosomes around promoters are more closely spaced than in bulk chromatin (Supplementary Fig. 1A). A complex of $\sim 100 \mathrm{bp}$, located downstream of the TSS, is also visible in the Pol II fragMaps. Possible origins for this unanticipated complex will be discussed later. Divergent transcription occurs at variable distances upstream of the sense TSS on the host genome, so the upstream region displays a similar, but less well-defined pattern compared to transcription in the sense direction. Pol II and H3K4me3 fragMaps from Exp 3 datasets displayed all the same features and were virtually indistinguishable from those using Exp4 datasets (Supplementary Fig. 3D) demonstrating that the method is robust and reproducible.

Major differences from the host patterns were found when analyzing the HCMV genome. The H3K4me3 signal was not positioned around the majority of promoters (Fig. 3D). HCMV Pol II fragMaps confirm the pervasiveness of Pol II transcription with Pol II visible across the fragMap (Fig. 3D). The TSRs utilized to generate HCMV fragMaps are 200 bp so individual TSRs may be represented multiple times in the $2000 \mathrm{bp}$ window. This causes a light background of particularly sized fragments across the entire visualized region. The fragments near the TSS show free paused Pol II, Pol II positioned over the TSS in $~ 75$ bp fragments, and an additional complex of $\sim 50$ bp positioned just upstream of the TSS that is not present on host fragMaps. This $~ 50$ bp protection over the TSS is especially evident in Exp3 viral fragMaps (Supplementary Fig. 3E). Nucleosome-abutted Pol II, which is the most abundant feature seen on the host genome, is present but at a vastly lower level than free Pol II on the HCMV genome.

The apparent differences in Pol II association with the +1 nucleosome on the host and HCMV genomes prompted us to quantify how frequently Pol II encounters an immediately downstream nucleosome in these two cases. The number of reads in features corresponding to the free Pol II, abutted Pol II, and +1 nucleosome based on genomic position of fragment centers and fragment size were quantified from Pol II and H3K4me3 datasets (Supplemental Excel file). Analysis of free or abutted Pol II demonstrated that $36 \%$ of the total engaged Pol II signal arises from free Pol II on the host genome. In contrast to the host, $74 \%$ of the engaged Pol II was free on the HCMV genome. On a promoter by promoter basis, $85 \%$ have more free than abutted Pol II signal on the HCMV genome, whereas only $18 \%$ do on host (Fig. 3E). Examination of the fragment count of +1 nucleosome sized fragments from the H3K4me3 dataset shows that the host promoters with the absolute highest percentage of free Pol II have little +1 nucleosome, but most promoters have a similar amount of +1 nucleosome (Fig. 3E). Although the H3K4me3 signal over the viral genome has a similar value in terms of reads, there are about a hundred times more viral genomes and thus the H3K4me3 modified nucleosome occupancy over the viral genomes is about $1 \%$ of that in the host. 


\section{Detection and characterization of TBP-driven PICs and UL87-driven viral PICs}

The existence of Pol II-containing complexes that extended upstream of the MaxTSS strongly suggested that these features correspond to PICs, so Pol II DFF-ChIP was repeated under conditions that would alter PIC prevalence. Treatment of cells for an hour with $1 \mu \mathrm{M}$ triptolide, an inhibitor of transcription initiation, should increase the PIC relative to paused Pol II and that is exactly what was found for the $\sim 75$ bp feature on the host and viral genomes (Fig. 4A). Triptolide treatment also more clearly reveals the $\sim 50 \mathrm{bp}$ feature directly upstream of the TSS, which is unique to the viral genome (Fig. 4A). As an additional verification that $\sim 75 \mathrm{bp}$ protections arose from uninitiated Pol II, Exp1 and Exp2 DFF-ChIP were reanalyzed since they utilized different wash conditions in a GFP-Pol II tagged MRC5 cell line ${ }^{30}$ and GFP nanobody beads. The immunoprecipitations in these experiments were carried out with $150 \mathrm{mM}$ or $1 \mathrm{M}$ salt wash conditions. The $\sim 75$ bp feature was preferentially lost during the high salt conditions as expected ${ }^{33}$ for uninitiated Pol II (Supplementary Fig. 4). DFF-ChIP was then performed targeting the TATA-binding protein (TBP), a critical component of the host PIC. FragMaps generated from the TBP dataset show primarily the $\sim 75$ bp feature (Fig. 3A), indicating that these complexes are indeed TBP-containing PICs. Additionally, TBP fragMaps reveal protections that share the relatively sharp upstream edge with the full TBP PIC but are much smaller in size, about $40 \mathrm{bp}$. These likely result from TBP-containing complexes prior to incorporation of Pol II and assembly of the complete PIC. DFF-ChIP was also performed utilizing a Pol II antibody that targets the serine 5 phosphorylation on the CTD of Pol II (Ser5P). Ser5P modification is carried out by CDK7, a component of the initiation factor TFIIH ${ }^{34}$. The resulting fragMaps demonstrate that Ser5P antibodies recognize modified Pol II in the TBP PIC on both the host and viral genome. However, the $\sim 50$ bp PICs on the viral genome were not detected (Fig. 4B) suggesting that the Pol II in those complexes is not phosphorylated. There is a difference in the relative amounts of PIC and free paused Pol II detected with the F12 antibody and the Ser5P antibody. The epitope on the Pol II large subunit recognized by F12 is located deep within the PIC ${ }^{13}$ and potentially masked by TFIIE and TFIIH, while the Ser5P epitope would be difficult to mask because of its repetitive occurrence and disordered structure. Therefore, we favor the idea that the Ser5P results are more representative of Pol II levels in all TBP-driven promoter-proximal complexes. Supporting this idea, when MRC5 cells containing a GFP-tagged Pol II were immunoprecipitated with GFP nanobodies, the PIC was a major species (Supplementary Fig. 4B).

Large TBP fragMaps provided further insight into the chromatin differences between the host and HCMV genomes (Fig. 4C). Some TBP PICs on the host genome are associated with the +1 nucleosome, resulting in fragments of $\sim 250 \mathrm{bp}$. These interactions are mostly absent on the HCMV genome presumably because the viral genome is less chromatinized. Significant amounts of the host PICs driving divergent transcription also connect with the adjacent nucleosome (the -1 nucleosome), analogous to the PIC-nucleosome complexes in the sense direction. As with the free-standing PIC, the $\mathrm{PIC} /+1$ nucleosome feature on the host genome was salt sensitive (Supplementary Fig. 4C). Previous analysis of downstream sequences relative to TSSs revealed periodic elements that likely serve to position the +1 nucleosome ${ }^{6}$, suggesting a connection between the TSS and nucleosome positioning. Considering that both genic-oriented and divergent PICs are normally associated with well-positioned immediatelyadjacent nucleosomes, it is further likely that the PIC, TSS, and +1 nucleosome connection is important for specifying and/or facilitating transcription initiation.

Unlike the early HCMV transcriptional program that relies entirely on the host general Pol II transcription machinery, beta- and gamma herpesviruses have unique late promoters containing a TATT upstream element that recruits virally-encoded late transcription factors ${ }^{35,36}$. Since only one of the two distinct Pol II-containing PICs on the viral genome corresponds to the host complex driven by TBP, we posited that the 50 bp virus-specific PIC is based on UL87, one of the viral late transcription factors which associates with the TATT element ${ }^{17}$. DFF-ChIP was 
performed with a Towne strain of HCMV expressing a HA-tagged UL87 (Exp4). FragMaps demonstrated that the $\sim 50 \mathrm{bp}$ viral PICs were almost exclusively recovered with the HA antibody (Fig. 4D). Interestingly, unlike the 75 bp TBP-PICs the 50 bp UL87-PICs did not cover the TSS.

In an attempt to correlate upstream sequence elements with the extent of PIC assembly, UL87 PIC and TBP PIC features at each gene on the host or on each TSR of the HCMV genome were quantified (Supplementary Excel file). The size and position of these features were selected such that no overlap was allowed between them. Each region was then rank ordered by the amount of the UL87 PIC feature or the TBP PIC feature. Logos were generated by MEME analysis ${ }^{37}$ of the -38 to -19 region for the PICs in the top decile of occupancy on the host and viral genomes (Fig. 4E). Such analysis recapitulated the expected TA-rich binding motifs with TBP preferring sequences containing TATA and UL87 preferring sequences containing TATT. UL87 evidently has a stricter requirement for TATT containing sequences with 128/146 matches to its HCMV Logo, while TBP had a lower percentage of matches to its host (623/1230) and viral (88/146) Logos. It is important to note that not all host promoters with high levels of PIC occupancy are AT-rich in the -38 to -19 region ${ }^{28}$.

\section{TBP and UL87 are functionally distinct but not mutually exclusive on HCMV promoters}

To characterize the transcription of the HCMV genome throughout lytic infection and its relation to UL87 and TBP usage, PRO-Seq was carried out at multiple different time points of infection and compared to 48 hpi DFF-ChIP data. Time points include two early times (4 and 12 hpi), 24 hpi which is the beginning of replication, and two late times (48 and $72 \mathrm{hpi}$ ) in which high levels of viral replication have occurred and during which UL87 function is critical. Representative regions of the HCMV genome containing early and late genes are depicted in a 1,400 bp region and an 800 bp region with corresponding HCMV genomic fragMaps below showing fragments from the UL87, TBP, Pol II, and Ser5P datasets (Fig. 5A). The early promoter UL29 has only TBP, Pol II, and Ser5P fragments associated with it (Fig. 5A, blue TBP arrow), while the late intragenic promoter in UL49 only has UL87 and Pol II fragments (Fig. 5A, red UL87 arrow). TBP and UL87 driven promoters can occur very close to each other (Fig. 5A, red and blue Both arrows) or can even drive transcription from the exact same TSS (Fig. 5A, purple Both arrow). Overlapping PICs such as these would be expected to compete for occupancy on the HCMV genome. Quantification of 5' end reads found in an 11 bp window around the MaxTSS of these five promoters shows that TBP-driven TSSs are more active early in comparison to UL87 PICs that are active late (Supplementary Fig. 5A). Because some promoters are driven by both TBP and UL87 the distinction between early versus late gene transcription is more complex than a simple separation of TBP and UL87 driven promoters.

To quantify how TBP and UL87 usage is related to early or late expression, transcription from each promoter was quantified by counting $5^{\prime}$ ends in an $11 \mathrm{bp}$ window centered on the MaxTSS of each TSR at each time point. The fractional usage for each promoter at each time point was displayed in a heatmap after normalizing to the total number HCMV reads in each time point. 795 of the 1,461 promoters that each had a total of 100 reads across the time course were then sorted based on PFA sensitivity ${ }^{28}$, on a slope calculated from the 5 time points for each promoter and by UL87 dependency ${ }^{36}$ (Fig. 5B). Each promoter was colorized individually based on the relative usage across the time course. The three sorts gave similar patterns of genes with early (red to green) and late (green to red) transcription kinetics. Each TSR's reliance on DNA replication (PFA sensitivity) and UL87 dependency was then plotted against slope to classify how TSRs with primarily TBP (blue) and UL87 (red) PICs behaved in relation to time of expression (Supplementary Fig. 5B). The relative preference for TBP or UL87 PICs was calculated as the ratio of TBP/UL87 PICs after normalization of total counts in each feature. These plots show that UL87 primarily functions on genes with late transcription kinetics and that many of these promoters are also stimulated by DNA replication. However, and significantly, 
many promoters with late kinetics are primarily TBP driven showing that TBP is also essential in transcription of some late genes. The UL22A promoter has a TBP/UL87 ratio of 0.93 and displays late kinetics. There are two main TSSs at all time points that are separated by 3 bp with a shift in the relative usage at early and late time points that is reverted when viral replication is blocked by PFA treatment (Supplementary Fig. 5C). This promoter exemplifies a clear competition for formation of TBP and UL87 PICs.

Nearly all promoters show some level of UL87 and TBP usage and to determine to what extent promoters are shared, the TBP/UL87 PIC ratio was calculated for all 1,461 TSRs and plotted (Fig. 5C). The results indicate that the relative levels of UL87 and TBP PICs vary by many orders of magnitude across the 1461 TSRs. Therefore, in order to make comparisons between TBP and UL87 PICs only the top and bottom 5\% of TSRs sorted by PIC ratio were utilized to prevent contaminating signal from the other class of PIC. To compare initiation efficiency, fragMaps were generated utilizing the TBP and Ser5P datasets for the top TBP TSRs and UL87 and Pol II datasets for the top UL87 TSRs (Fig. 5D). As noted above, the F12 antibody epitope in the large subunit of Pol II is significantly masked in TBP PICs ${ }^{13}$, so the Ser5P Pol II signal was used to visualize Pol II in TBP driven TSRs. The strong correlation of feature counts from the Ser5P dataset in comparison to TBP and Pol II datasets also advocates for this approach (Supplementary Fig. 5D). These fragMaps reveal that UL87 and TBP driven TSRs yield similar amounts of engaged Pol II in relation to PIC amounts. This result was initially surprising given the prominence of UL87 PIC peaks in comparison to detected paused Pol II downstream of those PICs, which seemed to indicate poor UL87 initiation. However, it is possible that a significant fraction of $\sim 50 \mathrm{bp}$ UL87 features may not have Pol II associated with them and this is supported by the relative lack of protection downstream of the TSS. Critically, on both promoter classes the PIC is as prominent on chromatin as paused Pol II indicating that the PIC is far more prevalent on the genome than expected.

\section{Nucleosomes on the HCMV genome are irregularly spaced}

To directly assess if sites of initiation correlated with H3K4me3 modified nucleosomes, genome browser tracks and genomic fragMaps for TBP, UL87 and H3K4me3 were compared. There is little evidence for a correlation of sites of transcription initiation and $\mathrm{H} 3 \mathrm{~K} 4 \mathrm{me} 3$ modification regardless of PIC type (Fig. 6A). This comparison does allow for a rough estimation of the number of nucleosomes that span the 21,000 bp region shown, since approximately 70 80 nucleosomes are distinguishable. This indicates that on average a nucleosome is positioned every 250-300 bp on the HCMV genome. This average spacing is consistent across the entire HCMV genome (Supplementary Fig. 6). It is unclear what drives nucleosome positioning on HCMV DNA but given the scarcity of transcription-coupled nucleosomes it is unlikely to be related to transcription. Metaplot analysis of all 1,461 viral TSRs shows that nucleosomes around promoters are spaced approximately 250-300 bp in contrast a much more compact spacing around promoters on the host genome of about $150 \mathrm{bp}$ (Fig. 6B). A PIC containing TBP is capable of associating with the +1 nucleosome on the host (Fig. $4 \mathrm{C}$ ) and this prompted us to investigate if viral TSRs with strong TBP signal have a better positioned downstream nucleosome. Selecting the top $10 \%$ of TSRs with the highest level of TBP PICs showed that indeed these TSRs have a slightly stronger +1 nucleosome signal (Fig. 6C). The same analysis performed with the top UL87 TSRs failed to reveal any clear nucleosome patterning (Fig. 6C). These findings suggest that while TBP PICs may aid in the positioning of a +1 nucleosome, this is not a driving force for nucleosome positioning on the HCMV genome.

\section{Increasing the extent of DFF digestion reveals a general robustness of features and captures Pol II association with sub-nucleosomal fragments}

To confirm reproducibility and determine the effects of more extensive DFF digestion, five datasets were generated in duplicate using HFFs infected with the Towne strain of HCMV 
(Exp5). The datasets correlated highly within Exp5 and between Exp5 and Exp4 (Supplementary Fig. 7A). Total library fragment length distributions for Pol II, TBP, and H3K4me3 were compared between the initial and more extensively digested experiments (Fig. 7A and 7B). It was apparent that higher levels of digestion resulted in a division of some complexes into subgroups typically separated by a $10 \mathrm{bp}$ periodicity. Over-digestion did not change the ratio of the free to abutted Pol II (Fig. 7C and 7D), but the group of Pol II-containing fragments of $\sim 90$ to 110 bp became more apparent after increased digestion. Connections of the TBP PIC to downstream nucleosomes were mostly lost with over-digestion.

The H3K4me3 fragment distributions in Fig 7 clearly show that DFF can cleave within nucleosomes at higher digestion levels. Two classes of products shorter than the expected protection from the full nucleosome were observed: fragments which apparently resulted from removal of 10 or $20 \mathrm{bp}$ from the nucleosome ends and another population of centered fragments less than $80 \mathrm{bp}$ consistent with protection by the $\mathrm{H} 3 / \mathrm{H} 4$ tetramer. The lack of fragments between the two populations suggests that complete loss of the flanking $\mathrm{H} 2 \mathrm{~A} / \mathrm{H} 2 \mathrm{~B}$ dimers on the edges of the nucleosome occurs before the central $\mathrm{H} 3 / \mathrm{H} 4$ is invaded by DFF. All sub-nucleosomal fragments were centered including those less than $80 \mathrm{bp}$, further demonstrating that they arose from the H3/H4 tetramer (Supplementary Fig. 7C).

To better understand the interaction of Pol II with the +1 nucleosome we specifically considered well positioned nucleosomes downstream of the most focused promoters ${ }^{6}$ (Supplementary Fig. 7B). At such promoters, the upstream edge of the +1 nucleosome is positioned on average at +47 , just downstream of the average paused Pol II position at +41 . Pol II protects approximately 20 bp of DNA downstream of the active site ${ }^{38}$, suggesting that the leading edge of the abutted Pol II intrudes 1.5 DNA turns into the nucleosome. Pol II at this location would disrupt the $\mathrm{H} 3$ contact at nucleosome entry but could leave the $\mathrm{H} 2 \mathrm{~A} / \mathrm{H} 2 \mathrm{~B}$ contacts with DNA at least partially intact. If invasion by Pol II distorts the nucleosome, this could in turn reveal a cutting site for DFF downstream of the $\mathrm{H} 2 \mathrm{~A} / \mathrm{H} 2 \mathrm{~B}$ dimer. DFF cleavage in such a complex would give rise to the 87-107 bp fragment set that we observe in Pol II IPS, more prominently upon over-digestion (Fig. 7, and Supplementary Fig. 7). Other possible origins for this $\sim 100 \mathrm{bp}$ Pol II fragment set can be envisioned, which will be addressed in the Discussion.

\section{Discussion}

Our results provide a deeper understanding of transcription complexes and their interactions with chromatin on both the host and HCMV genomes. Critically, analysis of the length and position of fragments recovered by DFF-ChIP using fragMaps not only provided detailed footprints of PICs and paused Pol II but also directly revealed the interactions of those complexes with the +1 nucleosome. Using HCMV infected HFFs, we demonstrated that Pol II encounters a vastly different chromatin environment on the viral genome than it does on the host genome. Furthermore, our direct visualization of both TBP and UL87-driven PICs sheds light on sequence preferences, dimensions, and shared usage at the two promoter classes.

Our results provide a new perspective into the transcriptional processes during lytic infection in relation to the chromatin structure of the HCMV genome. Earlier work suggested that during productive infection, viral genomes form irregular nucleosome arrays ${ }^{21,39,40}$. However, a more recent genome wide analysis utilizing MNase suggested that the HCMV genome is largely packaged into nucleosomes throughout the viral cycle ${ }^{24}$. Our data confirm that nucleosomes are deposited across the viral genome at low level of occupancy, but we also show in multiple ways that paused Pol II rarely encounters a nucleosome on HCMV DNA. The TBP, Pol II, Ser5P, and UL87 DFF-ChIP signals corresponding to initiating Pol II or PICs often reside in the middle of apparently nucleosome rich regions on HCMV DNA despite the known ability of a nucleosome to block initiation ${ }^{31,32}$. Therefore, those regions of HCMV genomes with nucleosomes over TSSs cannot be transcriptionally active. Transcription complexes themselves report on local 
nucleosomes by conferring a larger protection footprint when they are in close proximity to a nucleosome. HCMV chromatin very rarely confers these protection patterns. Although it is likely that the large majority of HCMV promoters do not feature modified nucleosomes immediately downstream, it is not possible to prove that this is the case at every individual viral promoter. Post-translationally modified nucleosomes may be positioned on individual loci at some stages of the viral life cycle 22,23 . Overall, we conclude that during lytic infection the HCMV genome is transcribed in a predominantly nonchromatinized state.

The unique ability to recover PICs with DFF-ChIP without prior crosslinking was likely allowed by the use of EDTA during very rapid nuclei isolation ${ }^{41}$ prior to digestion and immunoprecipitation steps. EDTA was included to halt transcription but it may also increase PIC retention by eliminating the destabilization caused by ATP33 in abortive initiation and XPB function. Substantial recovery of PICs in DFF-ChIP has allowed a better understanding of their properties in the nucleus including documenting that TBP- and UL87-driven PICs are major features on the host (TBP) and viral genomes (TBP and UL87), equaling or surpassing paused Pol II in amount. The formation of the TBP-containing PIC requires Pol II in a hypophosphorylated state, but after PIC assembly in vitro, phosphorylation of the CTD may occur even prior to formation of the first phosphodiester bond ${ }^{42,43}$. Our data demonstrate that this actually occurs in cells, showing that Ser5P is present on TBP PICs (Fig. 3B). Additionally, we show that a substantial fraction of PICs directly interacts with the +1 nucleosome.

DFF-ChIP also uncovered differences between TBP and UL87 PICs and their interplay on the HCMV genome. TBP PIC footprints correspond to the known in vitro footprint of TFIID, from roughly 40 bp upstream of the TSS to 35 bp downstream ${ }^{6}$. Published human TFIID and PIC structures indicate that TFIID and XPB both contact DNA well downstream of the TSS ${ }^{2,44}$. In contrast, UL87 PIC footprints are only located upstream of the TSS, suggesting that UL87 PICs lack the subunits of TFIID that contact downstream DNA and crucially, at least the XPB subunit of TFIIH. Since UL87 PICs also lack Ser5P modification, it seems likely that the UL87 PICs we detect lack TFIIH. However, initiation at UL87 PICs is sensitive to inhibition of XPB by triptolide. We therefore propose that while TFIIH is required for UL87 initiation, it is not a stable component of the UL87 PIC that we detect. A recent study regarding ORF24, a UL87 homolog in Kaposi's sarcoma associated virus ${ }^{20}$, showed association with the Pol II CTD only in the hypophosphorylated state, which is in agreement with our data demonstrating that the Pol II in UL87 PIC is predominantly unphosphorylated ${ }^{45}$. Regardless of these functional differences, both PICs function on a large shared subset of HCMV promoters.

We expected from our earlier study that paused Pol II complexes would be located upstream of the +1 nucleosome or abutted to that nucleosome ${ }^{6}$. Those complexes are evident from DFF-ChIP (Fig. 2C) but high levels of DFF digestion in particular revealed unanticipated Pol II-containing fragments of 87-107 bp that extend downstream into the region that is expected to be protected by the proximal $\mathrm{H} 2 \mathrm{~A} / \mathrm{H} 2 \mathrm{~B}$ dimer of the +1 nucleosome (Fig. S4D). We suggested above that polymerase invasion of the nucleosome could reveal a site for DFF cleavage downstream of the dimer. However, other recent work suggests a plausible alternative explanation. It was reported that the Chd1 chromatin remodeler associates with +1 nucleosomes, specifically on the promoter-proximal face ${ }^{46}$. Subsequent structural studies showed that in a Chd1-nucleosome complex, a Chd1 domain displaces DNA from the nucleosome surface normally occupied by $\mathrm{H} 2 \mathrm{~A} / \mathrm{H}_{2} \mathrm{~B}^{47}$. Thus, the 87-107 bp Pol II-containing fragments we detected could have arisen from Pol II paused at the entry of a +1 nucleosome already occupied by Chd1. Presumably the interface of Chd1 and the nucleosome is more easily accessible at high levels of DFF digestion. In the earlier studies it was speculated that displacement of the Chd1 domain at nucleosome entry by the advancing polymerase would activate the remainder of Chd1 to drive displacement of the nucleosome and thus facilitate traversal by Pol II. Thus, this model predicts that once Pol II has displaced the proximal Chd1 domain, full traversal of the +1 nucleosome should be efficient ${ }^{47}$. This is consistent with the fact 
that we did not detect Pol II-containing complexes by DFF-ChIP corresponding to pausing just upstream of $\mathrm{H} 3 / \mathrm{H} 4$ tetramer of the +1 nucleosome, as might have been predicted from earlier in vitro studies ${ }^{48}$. Other results based on micrococcal nuclease digestion patterns indicated that in Drosophila +1 nucleosomes frequently lack the proximal H2A/H2B dimer ${ }^{49}$. However, as just noted, we do not have evidence from our experiments for a Pol II barrier at the $\mathrm{H} 3 / \mathrm{H} 4$ tetramer of the +1 nucleosome.

Future usage of DFF including DFF-ChIP holds great potential in uncovering intricacies of chromatin architecture. Targeted immunoprecipitation of various modified histones or histone replacements are but a few of the possibilities for these investigations. For direct inquiries into transcription, further DFF-ChIP experiments that target the general transcription factors involved in initiation, pausing, and productive elongation are of great interest and will aid in uncovering the ways that transcription complexes interact with nucleosomes. In addition, we expect DFFChIP to be applicable to the targeted investigation of many more specific chromatin associated factors.

\section{Methods}

\section{Viruses}

HCMV TB40/E BAC4 and Towne UL87HA were used in this study. The construction and use of the Towne UL87-HA recombinant virus was described previously ${ }^{28}$.

\section{Infections and treatments}

Primary human foreskin fibroblasts were maintained in Minimum Essential Medium (Gibco, 11095080 ) supplemented with $5 \%$ fetal bovine serum (Gibco, 26140079) and 1\% penicillinstreptomycin (Gibco, 15140122). Confluent (contact inhibited) HFF monolayers in T-150 cm² flasks were used for these studies. The culture medium was refreshed $24 \mathrm{~h}$ prior to infection. On the day of infection, all but $12 \mathrm{~mL}$ of the conditioned medium was removed and set aside. The remaining $12 \mathrm{~mL}$ of medium was inoculated with HCMV at a multiplicity of infection of 3 infectious units per cell (MOI of 3). Viral adsorption was carried out for $90 \mathrm{~min}$. The medium containing viral inoculum was then replaced with $12 \mathrm{~mL}$ of the conditioned medium. For experiments involving treatment with flavopiridol (Flavo; final concentration, $1 \mu \mathrm{M}$ ), or triptolide (final concentration, $1 \mu \mathrm{M}$ ), $6 \mathrm{~mL}$ of conditioned medium was temporarily removed $1 \mathrm{~h}$ before cells were harvested. This medium was treated with $6 \mu \mathrm{L}$ of $2 \mathrm{mM}$ Flavo (NIH AIDS Reagent Program 9925z) in DMSO, $6 \mu \mathrm{L}$ of $2 \mathrm{mM}$ triptolide (Sigma, T3652) in DMSO or $6 \mu \mathrm{L}$ of DMSO alone. Once inoculated with drug, the $6 \mathrm{~mL}$ of medium was immediately returned to the flask for a final $12 \mathrm{~mL}$ of culture medium. At $48 \mathrm{~h}$ post-infection, cells were lysed and cell nuclei were isolated and held in frozen storage until use, as described previously ${ }^{28}$.

\section{DFF-ChIP Seq}

DFF was purified as previously described 6 . For Exp4 approximately 6 million nuclei from human foreskin fibroblasts infected for 48 hours with human cytomegalovirus strains TB40/E or a mutant Towne virus expressing UL87-HA were digested with approximately $15 \mu \mathrm{g}$ of DFF in 20 $\mathrm{mM}$ HEPES (pH 7.6), $5 \mathrm{mM}$ magnesium acetate, $100 \mathrm{mM} \mathrm{K}(\mathrm{Ac}), 5 \mathrm{mM}$ DTT, for 1 hour at $37^{\circ} \mathrm{C}$. Digestions were carried out in batch where possible for all nuclei of the same treatments conserving the ratio of nuclei to DFF. Digestion was halted with the addition EDTA to a concentration four times that of magnesium and nuclei were subsequently split for individual IPs. Nuclei were lightly sonicated for $20 \mathrm{~s}$ at 40\% amplitude using Qsonica Q800R3 Sonicator and the supernatant was collected and brought up to $1 \mathrm{~mL}$ with solution containing $10 \mathrm{mM}$ Tris (pH 7.5), $100 \mathrm{mM} \mathrm{NaCl}, 1 \mathrm{mM}$ EDTA, and TritonX-100 such that the final concentration was $0.1 \%$. The supernatants were precleared for 20 minutes over Protein A (Sigma P9424) or G Sepharose (Sigma P3296) beads. Afterwards, the supernatants were removed from beads and immunoprecipitated with approximately $2.5 \mu \mathrm{g}$ of antibodies for Pol II (Santa Cruz, sc-55492), 
TBP (Abcam, ab51841), Ser5P (Millipore, 3E8), HA-tag (Cell Signaling Technology, C29F4), and $\mathrm{H} 3 \mathrm{~K} 4 \mathrm{me} 3$ (Abcam, ab8580) overnight at $4^{\circ} \mathrm{C}$ with rotation. Next, samples were incubated with Protein A beads (Protein G beads for Ser5P IPs) for 2 hours at $4^{\circ} \mathrm{C}$ with rotation. The beads were than washed five times with $10 \mathrm{mM}$ Tris ( $\mathrm{pH} 7.5), 150 \mathrm{mM} \mathrm{NaCl}, 1 \mathrm{mM}$ EDTA, and $0.1 \%$ TritonX for five minutes per wash. Bound material was than eluted twice with $50 \mu \mathrm{L}$ of 10 $\mathrm{mM}$ Tris (pH 7.5), $1 \% \mathrm{SDS}$, and $1 \mathrm{mM}$ EDTA incubated at $65^{\circ} \mathrm{C}$ for 5 minutes. Eluted material was subsequently treated with $20 \mu \mathrm{g}$ RNAse $A$ for 30 minutes at $37^{\circ} \mathrm{C}$ and then $40 \mu \mathrm{g}$ of Proteinase $\mathrm{K}$ for 2 hours at $65^{\circ} \mathrm{C}$. The same protocol was used for the all DFF-ChIP experiments with slight modifications. For MRC-5 expressing GFP-Pol II and HeLa cell lines in Exp1 and Exp2, 12 million nuclei were digested with $30 \mu \mathrm{g}$. After splitting and preclearing on Protein A sepharose beads, GFP-Pol II samples were incubated with Chromotek GFP-Trap beads for 4 hours, washed five times with either the same buffer as above or one containing $1 \mathrm{M}$ $\mathrm{NaCl}$ for $\sim 1$ min each, and eluted as described above. Samples from HeLa cells were immunoprecipitated, eluted, and treated the same as Exp4 except with $\sim 1$ min washes. Exp3 was performed exactly as Exp4 was done, but again with $\sim 1$ min washes. For Exp5 utilizing HFFs infected with Towne, 3 million HFF cells infected with Towne UL87-HA were incubated with approximately $25 \mu \mathrm{g}$ DFF. All libraries were cleaned up utilizing Invitrogen PureLink PCR purification kit. All libraries were prepared using the KAPA Hyper Prep Kit according to their protocol (Roche 7962312001). For libraries in experiments 1 and 2 we utilized Illumina TruSeq adapters. For all subsequent libraries, we created custom adapters utilizing the TruSeq sequences that contained an 8 bp UMI immediately downstream of the index. Test amplifications were performed on each library to determine the number of cycles necessary to obtain enough library material for sequencing. Full-scale amplification was then performed, fragments were quantified using an Agilent Bioanalyzer 2100, and then libraries were pooled and size selected from 135-1000 bp using a BluePippin. Prior to submission, proper size selection was confirmed with reanalysis using the Agilent Bioanalyzer. Libraries were sequenced on either an Illumina HiSeq 4000 or a NovaSeq 6000 by the lowa Institute of Human Genetics.

\section{PRO-Seq and PRO-Cap}

PRO-Seq and PRO-Cap libraries were prepared as previously described ${ }^{28}$, with a few modifications. Frozen nuclei isolated from HFF infected with HCMV TB40/E (MOI 3) for 48 or 72 $\mathrm{h}$ were thawed on ice, gently pelleted, and resuspended in $40 \mu \mathrm{L}$ of a nuclear run-on buffer (20 mM HEPES ( $\mathrm{pH}$ 7.6), $5 \mathrm{mM}$ magnesium chloride, $100 \mathrm{mM}$ potassium chloride, $5 \mathrm{mM}$

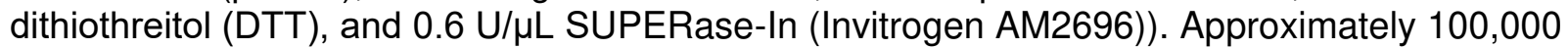
moth Sf21 nuclei were spiked into HFF nuclei prior to pelleting. Nuclei were warmed to $37^{\circ} \mathrm{C}$ and then combined with $20 \mu \mathrm{L}$ of a $3 X$ nuclear run-on mix (20 mM HEPES (pH 7.6), $5 \mathrm{mM}$ magnesium chloride, $100 \mathrm{mM}$ potassium chloride, $5 \mathrm{mM}$ DTT, 1.5\% Sarkosyl, and $60 \mathrm{uM}$ biotinylated ATP, UTP, GTP, and CTP (Perkin Elmer NEL544, NEL543, NEL545, and NEL542, respectively)). Samples were pulse-vortexed after the addition of nuclear run-on mix and incubated at $37^{\circ} \mathrm{C}$ for 10 minutes. Reactions were quenched with $40 \mu \mathrm{L}$ of $50 \mathrm{mM}$ ethylenediaminetetraacetic acid (EDTA) and $300 \mu \mathrm{L}$ of Trizol LS (Ambion 10296028), and total RNA was extracted according to manufacturer protocol. All subsequent steps in PRO-Seq library preparation were carried out as previously described ${ }^{28}$. PRO-Cap library preparation followed a similar procedure, except excluded the RNA hydrolysis step and included RNA polyphosphatase and terminator exonuclease treatments to ensure the integrity of nascent RNA 5 ' end capture. A detailed description of the PRO-Cap protocol has been published ${ }^{25}$. Reversetranscribed libraries were PCR amplified for 11 (PRO-Seq) or 17 (PRO-Cap) cycles, subjected to analysis on an Agilent Bioanalyzer 2100, pooled in equimolar ratios, size-selected on a Sage Science Blue Pippen (BDF2010 cassette, 135-600 bp fragments selected), and sequenced on an Ilumina HiSeq 4000 with 150 bp paired-end reads at the University of lowa Genomics 
Division. Raw data were trimmed, mapped, deduplicated, and processed into tracks as previously described ${ }^{28}$.

\section{DFF-ChIP analysis}

Initial workup of data was performed using DNAfastqtoBigWig (https://github.com/PTEFb/DNAfastatoBigWig). This is a linux based, multi-thread capable, Next Generation Sequencing (NGS) data analysis program with a command line interface. It performs the standard NGS data processing steps including, downloading sequencing data from a given web server, trimming adapter sequences from the sequencing data, aligning the trimmed data to a given list of genomes, generating mapping statistics for the aligned data, deduplication of aligned fragments using their Unique Molecular Identifiers (UMI), and finally, generating tracks for each sample in bigwig format. The program automatically accomplishes the following steps. Raw sequences in Fastq format were downloaded from the lowa Institute of Human Genetics (IIHG) Genome Sequencing web server using wget command. Next, adapter sequences were trimmed from these sequences using trim_galore 0.6 (https://github.com/FelixKrueger/TrimGalore/releases/tag/0.6.6) while retaining only paired end trimmed sequences of at least $18 \mathrm{bp}$ in size. These sequences were aligned with UCSC hg38, and Genbank TB40/E and Towne HCMV assemblies using bowtie v1.2.2 to generate alignments in sam format. UMls reads were used to deduplicate the aligned reads which were then converted into bed files. Unstranded tracks were generated for each sample by first converting bed into bedGraph format using bedtools v2.26, and subsequently into bigwig format using the Kent UCSC utility program called bedGraphToBigWig. All datasets are described in the supplementary Excel file. Raw and processed sequencing data can be obtained from GEO (GSEXXXX). All tracks can be viewed using the following links:

HOST: https://genome.ucsc.edu/s/David\%20Price/Host

Towne: https://genome.ucsc.edu/s/David\%20Price/Towne

TB40/E: https://genome.ucsc.edu/s/David\%20Price/TB40 E

\section{Fragment distribution plots}

Fragment size frequencies were calculated by counting the number of times a single fragment size or a range of fragment sizes are present in a given sample or overlap to a list of genomic intervals of a specific size. Bedtools v2.26 intersect program was used to generate overlap data between fragment and genomic intervals. Bash and awk scripts were used to generate counts for a single or a range of fragment sizes. Next, fragment sizes and their associated counts were sorted from short to long order. Additionally, fragment size counts were normalized to generate their relative amounts by dividing them with the total number of fragments present in a sample. MS Excel was used to plot relative and absolute counts of fragment sizes for each sample using scatter with straight lines option.

\section{Average base distribution plots}

The number of times a nucleotide is present at each position within a specified genomic interval was calculated as follows. Bedtools v2.26 getfasta program was used to generate FASTA files while maintaining same strand orientation for $\pm 100 \mathrm{bp}$ genomic intervals centered on fragment starts and ends. For these analyses all intervals centered on fragment starts were marked positive strand and intervals centered on fragment ends were marked negative strand. A custom linux based, multi-thread capable python script called ABD.py (https://github.com/P-TEFb/ABD) was run to generate absolute counts for each nucleotide across the given genomic intervals. Absolute nucleotide counts were converted to fractions by dividing them with the total number of sequences. MS Excel was used to plot base fractions across the genomic interval using scatter with straight lines option. Finally, the following color code was used for these plots: A was blue 
(hex code \#2222ff), T was orange (hex code \#ff6600), G was gray (hex code \#bbbbbb), and C was yellow (hex code \#dddd00).

\section{MaxTSSs analysis}

The truQuant program ${ }^{28}$ with an updated list of black listed regions was run on the PRO-Cap data (GSE113394) ${ }^{25}$ generated from uninfected HFF cells and DMSO bound NAS-Cap data $\left(\right.$ GSE139237) ${ }^{6}$ generated from HeLa cells using published parameters to generate host specific MaxTSSs. 12,229 (PRO-Cap) and 12,201 (NasCap) MaxTSSs associated with known host genes were used for further analysis. TsrFinderM2 ${ }^{6}$ was run on PRO-Cap datasets from TB40/E infected HFFs 72 hpi (GSEXXXX) and Towne infected HFFs 96 hpi (GSE113394) using published parameters to generate HMCV specific MaxTSSs. MaxTSSs associated with RNA 4.9 and without a \pm 1000 bp genomic sequence were excluded from further analysis. 1,461 TB40/E and 1,456 Towne MaxTSSs were used for further analysis.

\section{Feature analysis}

Feature analysis was performed by only counting fragments of a specific length whose center lay within a specific genomic interval using Bedtools v2.26 intersect program and simple awk commands for each MaxTSSs in the host and HMCV (TB40/E and Towne) genomes. The features consisted of fragments $30-65$ bp with a fragment center between +10 and +45 for free Pol II, 140-205 bp with a fragment center between +65 and +140 for abutted Pol II, 120-175 with a fragment center between +65 and +175 for +1 nucleosomes, $64-88$ with a fragment center between -18 and -2 for TBP PICs, and 40-63 with a fragment center between -36 and -18 for UL87 PICs. The engaged Pol II feature was found by summing the free Pol II and abutted Pol II counts. Quantification of percentage of Pol II that was either abutted or free was done by selecting promoters with at least one read in either the abutted or free features and that have a MaxTSS of at least 10. Percentage of free Pol II was found relative to engaged Pol II for the whole dataset as well as a gene by gene basis. Quantification of PIC amounts to engaged Pol II was done by first normalizing the TBP PIC fragments from the TBP dataset and UL87 PIC fragments from the UL87 dataset. The ratio between these normalized values was used to rank order promoters by UL87 or TBP dominance and then the ratio of PIC to engaged Pol II was calculated. The Ser5P dataset was utilized for analysis of TBP PICs.

\section{FragMaps}

Heatmaps displaying the average distribution and position of fragments from DFF-Seq and DFF-ChIP across individual genome intervals or collections of intervals were created using fragMap.py (https://github.com/P-TEFb/fragMap). In general, collections were centered on the MaxTSSs from truQuant annotations that provide the most highly utilized TSS for each active gene with proper strand orientation. In some cases, subsets of the truQuant annotations were used. The data was generated by counting the total number of fragments of each fragment size between 18 and 400 bp across the genomic interval. The aspect ratio, number of pixels, intensities assigned, and the shape of major and minor tick marks were controlled. The desired aspect ratio was implemented by choosing a discrete number of pixels for each base or fragment size. The values at each horizontal position of the fragment sizes were used to assign intensities using the gray.colors function in $\mathrm{R}$ with 0 being white and maximum being black. $A$ linear relationship between relative read value and intensity was utilized. Black was set at the maximum read value for most frag maps. To correct for human inaccuracies in perception of dark and light patterns on heatmaps, a gamma correction of 0.5 was applied to all fragMaps.

\section{Statistics}

Pearson correlation coefficient " $r$ " was calculated to demonstrate the reproducibility of our datasets using the MS excel CORREL function. Pearson's $r$ computes the effect of change in 
one variable compared to the change in another variable. MEME motif discovery tool was used to short sequence motifs in a set of longer DNA sequences. Motifs with an E-value less than 0.05 were considered as significantly enriched in our dataset. E-value is an estimation of seeing similar motifs of identical width and contributing sites in a similar size dataset of random sequences.

\section{Acknowledgments}

We thank J. A. Marteijn for providing MRC5 cells expressing GFP-tagged Pol II. This research was supported by the National Institute of General Medical Sciences (grant GM126908 to D.H.P. and GM121428 to D.S.L.) and the Department of Veterans Affairs (merit award 1BX001107 to J.L.M.).

\section{Author Contributions}

BMS developed the DFF-ChIP protocol, performed all DFF-ChIP experiments, analyzed the data and wrote the paper. MP performed all bioinformatics. ML and JLM generated the HCMV infected HFFs. CBB performed the HCMV time course PRO-Seq experiment. DSL provided intellectual input throughout the study and helped refine the manuscript. DHP directed the research and helped create the bioinformatics approaches.

\section{Competing Interests}

No authors have competing interests. 


\section{References}

1. Compe, E. \& Egly, J.M. The Long Road to Understanding RNAPII Transcription Initiation and Related Syndromes. Annu Rev Biochem 90, 193-219 (2021).

2. Patel, A.B., Greber, B.J. \& Nogales, E. Recent insights into the structure of TFIID, its assembly, and its binding to core promoter. Curr Opin Struct Biol 61, 17-24 (2020).

3. Weber, C.M., Ramachandran, S. \& Henikoff, S. Nucleosomes are context-specific, H2A.Z-modulated barriers to RNA polymerase. Mol Cell 53, 819-30 (2014).

4. Santos-Rosa, H. et al. Active genes are tri-methylated at K4 of histone H3. Nature 419, 407-11 (2002).

5. Howe, F.S., Fischl, H., Murray, S.C. \& Mellor, J. Is H3K4me3 instructive for transcription activation? Bioessays 39, 1-12 (2017).

6. Luse, D.S., Parida, M., Spector, B.M., Nilson, K.A. \& Price, D.H. A unified view of the sequence and functional organization of the human RNA polymerase II promoter. Nucleic Acids Res 48, 7767-7785 (2020).

7. Vermeulen, M. et al. Selective anchoring of TFIID to nucleosomes by trimethylation of histone H3 lysine 4. Cell 131, 58-69 (2007).

8. Lauberth, S.M. et al. H3K4me3 interactions with TAF3 regulate preinitiation complex assembly and selective gene activation. Cell 152, 1021-36 (2013).

9. Day, D.S. et al. Comprehensive analysis of promoter-proximal RNA polymerase II pausing across mammalian cell types. Genome Biol 17, 120 (2016).

10. Jimeno-Gonzalez, S., Ceballos-Chavez, M. \& Reyes, J.C. A positioned +1 nucleosome enhances promoter-proximal pausing. Nucleic Acids Res 43, 3068-78 (2015).

11. Verger, A., Monte, D. \& Villeret, V. Take Your PIC. Trends Biochem Sci (2021).

12. Chen, $X$. et al. Structural insights into preinitiation complex assembly on core promoters. Science 372(2021).

13. Abdella, R. et al. Structure of the human Mediator-bound transcription preinitiation complex. Science (2021).

14. Aibara, S., Schilbach, S. \& Cramer, P. Structures of mammalian RNA polymerase II preinitiation complexes. Nature 594, 124-128 (2021).

15. Meier, J.L. \& Stinski, M.F. Major Immediate-Early Enhancer and Its Gene Products. Cytomegaloviruses: From Molecular Pathogenesis to Intervention, Vol I, 152-173 (2013).

16. Paulus, C. \& Nevels, M. The human cytomegalovirus major immediate-early proteins as antagonists of intrinsic and innate antiviral host responses. Viruses 1, 760-79 (2009).

17. Wyrwicz, L.S. \& Rychlewski, L. Identification of Herpes TATT-binding protein. Antiviral Res 75, 167-72 (2007).

18. Aubry, V. et al. Epstein-Barr virus late gene transcription depends on the assembly of a virus-specific preinitiation complex. J Virol 88, 12825-38 (2014).

19. Gruffat, H., Kadjouf, F., Mariame, B. \& Manet, E. The Epstein-Barr virus BcRF1 gene product is a TBP-like protein with an essential role in late gene expression. $J$ Virol 86 , 6023-32 (2012).

20. Davis, Z.H. et al. Global mapping of herpesvirus-host protein complexes reveals a transcription strategy for late genes. Mol Cell 57, 349-60 (2015).

21. Nitzsche, A., Paulus, C. \& Nevels, M. Temporal dynamics of cytomegalovirus chromatin assembly in productively infected human cells. $J$ Virol 82, 11167-80 (2008).

22. Cuevas-Bennett, C. \& Shenk, T. Dynamic histone H3 acetylation and methylation at human cytomegalovirus promoters during replication in fibroblasts. $J$ Virol 82, 9525-36 (2008).

23. Groves, I.J., Reeves, M.B. \& Sinclair, J.H. Lytic infection of permissive cells with human cytomegalovirus is regulated by an intrinsic 'pre-immediate-early' repression of viral gene expression mediated by histone post-translational modification. J Gen Virol 90 , 2364-2374 (2009). 
24. Zalckvar, E. et al. Nucleosome maps of the human cytomegalovirus genome reveal a temporal switch in chromatin organization linked to a major IE protein. Proc Natl Acad Sci U S A 110, 13126-31 (2013).

25. Parida, M. et al. Nucleotide Resolution Comparison of Transcription of Human Cytomegalovirus and Host Genomes Reveals Universal Use of RNA Polymerase II Elongation Control Driven by Dissimilar Core Promoter Elements. mBio 10(2019).

26. Griffiths, P. \& Reeves, M. Pathogenesis of human cytomegalovirus in the immunocompromised host. Nat Rev Microbiol (2021).

27. Britt, W.J. Maternal Immunity and the Natural History of Congenital Human Cytomegalovirus Infection. Viruses 10(2018).

28. Li, M. et al. Human cytomegalovirus IE2 drives transcription initiation from a select subset of late infection viral promoters by host RNA polymerase II. PLoS Pathog 16, e1008402 (2020).

29. Liu, X., Zou, H., Slaughter, C. \& Wang, X. DFF, a heterodimeric protein that functions downstream of caspase-3 to trigger DNA fragmentation during apoptosis. Cell 89, 17584 (1997).

30. Steurer, B. et al. Live-cell analysis of endogenous GFP-RPB1 uncovers rapid turnover of initiating and promoter-paused RNA Polymerase II. Proc Natl Acad Sci U S A 115, E4368-E4376 (2018).

31. Knezetic, J.A. \& Luse, D.S. The presence of nucleosomes on a DNA template prevents initiation by RNA polymerase II in vitro. Cell 45, 95-104 (1986).

32. Lorch, Y., LaPointe, J.W. \& Kornberg, R.D. Nucleosomes inhibit the initiation of transcription but allow chain elongation with the displacement of histones. Cell 49, 20310 (1987).

33. Cai, H. \& Luse, D.S. Transcription initiation by RNA polymerase II in vitro. Properties of preinitiation, initiation, and elongation complexes. J Biol Chem 262, 298-304 (1987).

34. Egly, J.M. \& Coin, F. A history of TFIIH: two decades of molecular biology on a pivotal transcription/repair factor. DNA Repair (Amst) 10, 714-21 (2011).

35. Gruffat, H., Marchione, R. \& Manet, E. Herpesvirus Late Gene Expression: A ViralSpecific Pre-initiation Complex Is Key. Front Microbiol 7, 869 (2016).

36. $\mathrm{Li}, \mathrm{M}$. et al. Cytomegalovirus late transcription factor target sequence diversity orchestrates viral early to late transcription. PLoS Pathog 17, e1009796 (2021).

37. Bailey, T.L. et al. MEME SUITE: tools for motif discovery and searching. Nucleic Acids Res 37, W202-8 (2009).

38. Samkurashvili, I. \& Luse, D.S. Translocation and transcriptional arrest during transcript elongation by RNA polymerase II. J Biol Chem 271, 23495-505 (1996).

39. Nevels, M., Nitzsche, A. \& Paulus, C. How to control an infectious bead string: nucleosome-based regulation and targeting of herpesvirus chromatin. Rev Med Virol 21, 154-80 (2011).

40. Kent, J.R. et al. During lytic infection herpes simplex virus type 1 is associated with histones bearing modifications that correlate with active transcription. $J$ Virol 78, 1017886 (2004).

41. Ball, C.B., Nilson, K.A. \& Price, D.H. Use of the nuclear walk-on methodology to determine sites of RNA polymerase II initiation and pausing and quantify nascent RNAs in cells. Methods 159-160, 165-176 (2019).

42. Kang, M.E. \& Dahmus, M.E. RNA polymerases IIA and IIO have distinct roles during transcription from the TATA-less murine dihydrofolate reductase promoter. J Biol Chem 268, 25033-40 (1993).

43. Laybourn, P.J. \& Dahmus, M.E. Phosphorylation of RNA polymerase IIA occurs subsequent to interaction with the promoter and before the initiation of transcription. $J$ Biol Chem 265, 13165-73 (1990). 
44. He, Y. et al. Near-atomic resolution visualization of human transcription promoter opening. Nature 533, 359-65 (2016).

45. Castaneda, A.F. et al. The gammaherpesviral TATA-box-binding protein directly interacts with the CTD of host RNA Pol II to direct late gene transcription. PLoS Pathog 16, e1008843 (2020).

46. Skene, P.J., Hernandez, A.E., Groudine, M. \& Henikoff, S. The nucleosomal barrier to promoter escape by RNA polymerase II is overcome by the chromatin remodeler Chd1. Elife 3, e02042 (2014).

47. Farnung, L., Vos, S.M., Wigge, C. \& Cramer, P. Nucleosome-Chd1 structure and implications for chromatin remodelling. Nature 550, 539-542 (2017).

48. Bondarenko, V.A. et al. Nucleosomes can form a polar barrier to transcript elongation by RNA polymerase II. Mol Cell 24, 469-79 (2006).

49. Ramachandran, S., Ahmad, K. \& Henikoff, S. Transcription and Remodeling Produce Asymmetrically Unwrapped Nucleosomal Intermediates. Mol Cell 68, 1038-1053 e4 (2017). 


\section{Figure Legends}

Figure 1. Reproducibility of Pol II and H3K4me3 DFF-ChIP

A Diagram of the DFF-ChIP method. Isolated nuclei are digested with DFF without crosslinking and lightly sonicated to release soluble DNA complexes. The soluble DNA is then immunoprecipitated, library prepped, and sequenced. B,C Genome browser tracks of Pol II DFF-ChIP (purple) and H3K4me3 DFF-ChIP (orange) from HeLa and MRC5 GFP-Pol II cells generated in two different experiments (Exp1 and Exp2). Browser tracks of Flavo NasCap PROSeq (black/grey) show transcription data. D Correlation plots of datasets from Exp1 and Exp2. Read counts in 10,000 bp windows around 12,229 HFF truQuant MaxTSSs were summed and plotted against sums from other experiments.

Figure 2. Genome browser tracks of H3K4me3 and Pol II DFF-ChIP from Infected HFFs A,B Genome browser tracks of PRO-Seq, Pol II DFF-ChIP, and H3K4me3 DFF-ChIP from HFFs 48 hpi on the hg38 and TB40/E genomes. C,D Genome browser tracks of PRO-Seq, Pol II DFF-ChIP, and H3K4me3 DFF-ChIP showing the GAPDH promoter in 5,000 and 1,000 bp windows. A dotted line denotes the TSS. E,F Genome browser tracks of PRO-Seq, Pol II DFFChIP, and H3K4me3 DFF-ChIP showing the an early (E) and late (F) promoter in a 1,000 bp windows. A dotted line denotes the TSS.

\section{Figure 3. Visualizing and quantifying transcription complexes and chromatin utilizing fragMaps}

A Length distribution of fragments +/- 1000 bp of each of 12,229 truQuant genes for the Pol II and H3K4me3 DFF-ChIP datasets from Exp4. B Fragment count of aligned fragments from Exp4 of specified length $+/-1000$ bp relative to the TSS of all 12,229 truQuant genes. Total fragment length counts were normalized. C fragMaps of H3K4me3 and Pol II for the 12,229 truQuant genes showing fragments from $18-400$ bp that are $+/-1000$ bp relative to the MaxTSS. A zoomed fragMap showing $18-120$ bp fragments that are $+/-100$ bp relative to the TSS is also shown (right). D fragMaps of H3K4me3 and Pol II for the 1,461 TSRs showing fragments from $18-400$ bp that are $+/-1000$ bp relative to the MaxTSS. A zoomed fragMap showing 18-120 bp fragments that are $+/-100$ bp relative to the TSS is also shown (right. Resulting fragMaps were lightened $100 \%$ to aid in visualization. E Quantification of percentage of the free Pol II feature signal relative to total Pol II feature signal (free + abutted) on both the host and TB40/E genomes on a gene by gene basis sorted by highest to lowest (Left). Fragment count of the Nuc1 feature signal from the H3K4me3 dataset on host (middle) and TB40/E (right) genomes utilizing the same sort.

Figure 4. Detection and characterization of TBP-driven PICs and UL87-driven viral PICs A,B fragMaps of fragments positioned +/- $100 \mathrm{bp}$ around the TSS that are 18-120 bp sized fragments. Host fragMaps were generated from 12,229 truQuant HFF promoters and HCMV (TB40/E) fragMaps were generated from 1,461 TSRs from the Pol II, Pol II + triptolide (Trp), TBP, and Ser5P DFF-ChIP datasets. A dotted line denotes the TSS. C fragMaps of fragments positioned $+/-1000 \mathrm{bp}$ around the MaxTSS that are 18-400 bp in size. The host fragMap was generated from 12,229 truQuant HFF promoters and HCMV (TB40/E) fragMap was generated from 1,461 TSRs using the TBP datasets. D UL87 fragMaps were generated from 1,456 Towne TSRs. A dotted line denotes the TSS. E LOGOs generated with MEME Suite 5.3.1 from the top $10 \%$ genes/TSRs with the most fragments present in the TBP PIC or UL87 PIC feature as detected by DFF-ChIP. Parameters were: ZOOPS, search only given strand, 1 motif, 6 bp motif. Fractions represent the number of sequences matching the sequence motif out of the number of input sequences. E values for the three LOGOs were: TBP host, 1.5e-398; TBP HCMV, 3.6e038; UL87 HCMV, 1.4e-082. 
Figure 5. Classification of TBP and UL87 usage on HCMV genes according to lateness A PRO-Seq tracks depicting $5^{\prime}$ ends of reads from HCMV infection time course including 4, 12 , 24,48 and 72 hpi datasets in 1,400 and 800 bp regions of the viral genome. Below are corresponding DFF-ChIP tracks and genomic fragMaps of the same region. B A set of 795 TSRs with greater than 100 MaxTSS $5^{\prime}$ ends (+/- 5bp) when all time points are summed were selected and sorted based on PFA sensitivity, slope, and UL87 dependency. Each TSR had each time point value normalized to library size and each TSR was colored independently. The time point with the highest relative transcription was colored green and lowest colored red. C Quantification of the relative usage of TBP and UL87. The amount of TBP PIC feature counted from the TBP dataset was normalized to the amount of UL87 PIC feature counted from the UL87 dataset for all 1,461 TSRs. The ratio of TBP PIC to UL87 PIC was used to sort the TSRs by TBP PIC dominance (High value) and then plotted. D FragMaps for the top 5\% TBP and UL87 dominated (C) TSRs utilizing 18-120 bp sized fragments positioned +/- 100 bp around the TSSs. Top TBP TSRs are depicted using TBP and Ser5P datasets whereas UL87 TSRs are depicted using UL87 and Pol II datasets.

Figure 6. Analysis of TBP and UL87 PICs on the HCMV genome A Genome browser tracks from H3K4me3, TBP, and UL87 datasets (Exp4) compared directly to genomic fragMaps of the same region of the HCMV genome. B Normalized metaplot of H3K4me3 signal around all 12,229 host truQuant promoters and 1,461 HCMV TSRs. The inner graph shows the HCMV metaplot with a different Y-axis. C Normalized metaplot of H3K4me3 signal around the top 10\% of HCMV TSRs determined by amount of TBP PIC feature or UL87 PIC feature.

Figure 7. FragMaps resulting from different extents of DFF digestion

A,B Total fragment distributions from Pol II, TBP, and H3K4me3 datasets from the control digestion condition (Exp4) and the excess digestion condition (Exp5). C,D Pol II, H3K4me3, and TBP fragMaps of +/- $1000 \mathrm{bp}$ around the TSS containing 18-400 bp fragments of 12,229 truQuant genes with either (C) control (Exp4) or (D) excess digestion (Exp5).

Figure S1. Analysis of DFF-Seq and comparison of DFF-Seq to MNase-Seq

A Fragment length distribution for the total DFF-Seq library and for +/- 1000 bp from 12,201 truQuant HeLa TSSs. Data was normalized such that total fragment counts were the same. Mono-, di-, and tri-nucleosome are demarcated. B Base distribution of the surrounding $60 \mathrm{bp}$ around the 5' ends DFF-Seq fragments.

Figure S2. Reproducibility of DFF-ChIP on infected HFFs

A Genome browser tracks DFF-ChIP H3K4me3 and Pol II tracks from Exp3 and Exp4 and 72 hpi PRO-Cap transcription data. Representative 8000 bp regions are shown on the host and HCMV genomes. B Correlations between Exp3 and Exp4 datasets. Reads from 10,000 bp windows centered on each host truQuant promoter or each viral $200 \mathrm{bp}$ TSR were summed and compared across datasets.

Figure S3. Visualizing transcription complexes and chromatin from DFF-Seq and Exp3 replicate data

A Length distribution of fragments $+/-1000$ bp of each of 12,229 truQuant genes for the Pol II and H3K4me3 DFF-ChIP Exp3 datasets. B Fragment count of aligned fragments of specified length +/- 1000 bp relative to the TSS of all 12,229 truQuant genes using Exp3 data. Total fragment length counts were normalized. C fragMap representation of DFF-Seq data shown using two different black values. D FragMaps of Exp3 H3K4me3 and Exp3 Pol II for the 12,229 
truQuant genes showing fragments from $18-400$ bp that are $+/-1000$ bp relative to the MaxTSS. A zoomed fragMap showing 18-120 bp fragments that are $+/-100 \mathrm{bp}$ relative to the TSS is also shown (right). E FragMaps of Exp3 H3K4me3 and Exp3 Pol II for the 1,461 TSRs showing fragments from $18-400 \mathrm{bp}$ that are $+/-1000 \mathrm{bp}$ relative to the MaxTSS. A zoomed fragMap showing 18-120 bp fragments that are $+/-100$ bp relative to the TSS is also shown (right).

Figure S4. DFF-Seq fragMap and salt sensitivity of TBP PICs on the host genome

A Genome browser tracks showing uninfected HFF PRO-Cap data and MRC5 Flavo GFP-Pol II DFF-ChIP tracks that were either washed with high or low salt as described in Methods. The dotted line demarcates the TSS. B fragMaps of $+/-1000$ bp around the TSS containing 18-400 bp sized fragments and zoomed in fragMaps of $+/-100$ bp around the TSS containing 18-120 bp sized fragments from 12,201 HeLa truQuant genes from the MRC5 GFP-Pol II datasets. Regions of free Pol II and PIC are demarcated by brackets. For the wider fragMaps, the black value was reduced 3 fold to emphasize smaller fragments. For the zoomed in fragMaps, max black levels were set based on the shown region.

\section{Figure S5. TBP PICs are functionally different from UL87 PICs even though both may appear at late promoters}

A Quantification of $5^{\prime}$ end reads in an $11 \mathrm{bp}$ window around the five TSSs specifically indicated in Fig. 5A. B A subset TSRs from Fig. 5B plotted with PFA sensitivity and UL87 dependency against slope. TSRs with a TBP/UL87 ratio greater than 2 were colored blue and TSRs with a ratio less than 0.5 were colored red. C Correlations of the amount of engaged Pol II and TBP PIC features counted utilizing the Ser5P dataset in comparison to the Pol II and TBP dataset, respectively, on the host and HCMV genomes. D Genome browser tracks depicting shifting 5' ends from PRO-Seq CMV infection time course data and DFF-ChIP tracks showing UL87, TBP, Pol II, and Ser5P datasets.

Figure S6. Genomic fragMaps spanning the entire HCMV genome for H3K4me3, TBP, and UL87 fragments

A Genomic fragMaps depicting overlapping 21,000 bp windows of the entire HCMV genome. H3K4me3 depict fragments between 18-400 bp whereas TBP and UL87 fragMaps depict fragments between 18-150 bp.

Figure S7. Characterization of fragments resulting from excess DFF digestion

A Correlation plots comparing number of reads in 10,000 bp windows around all 12,229 truQuant genes. Graphs compare Exp5 replicates and a single Exp5 replicate to Exp4 data. B H3K4me3 fragMaps from the top 25\% and bottom 25\% of the 12,229 truQuant genes sorted by TSS focus (standard deviation of TSSs in TSRs; Exp4). $\mathbf{C}$ fragMaps from the H3K4me3 and Pol II excess digestion experiments (Exp5) with schematic depicting likely nucleosome protections and DFF cleavage sites. 


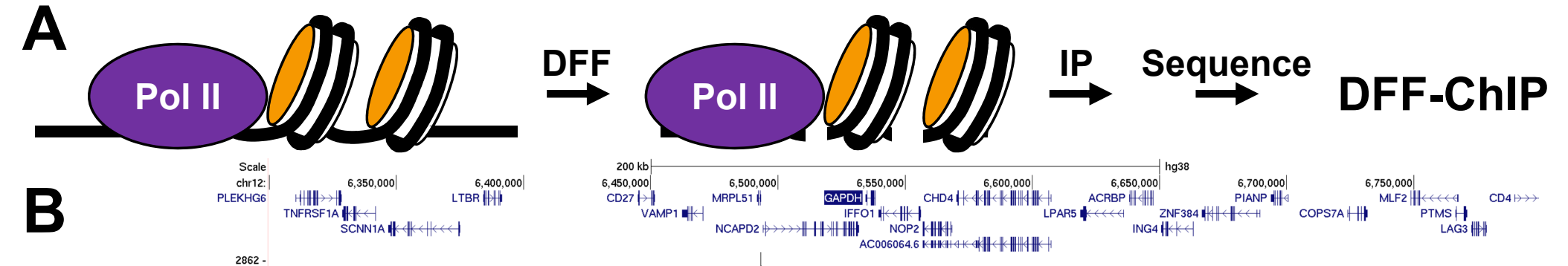

HeLa NasCap Flavo Fw

HeLa NasCap Flavo Rv
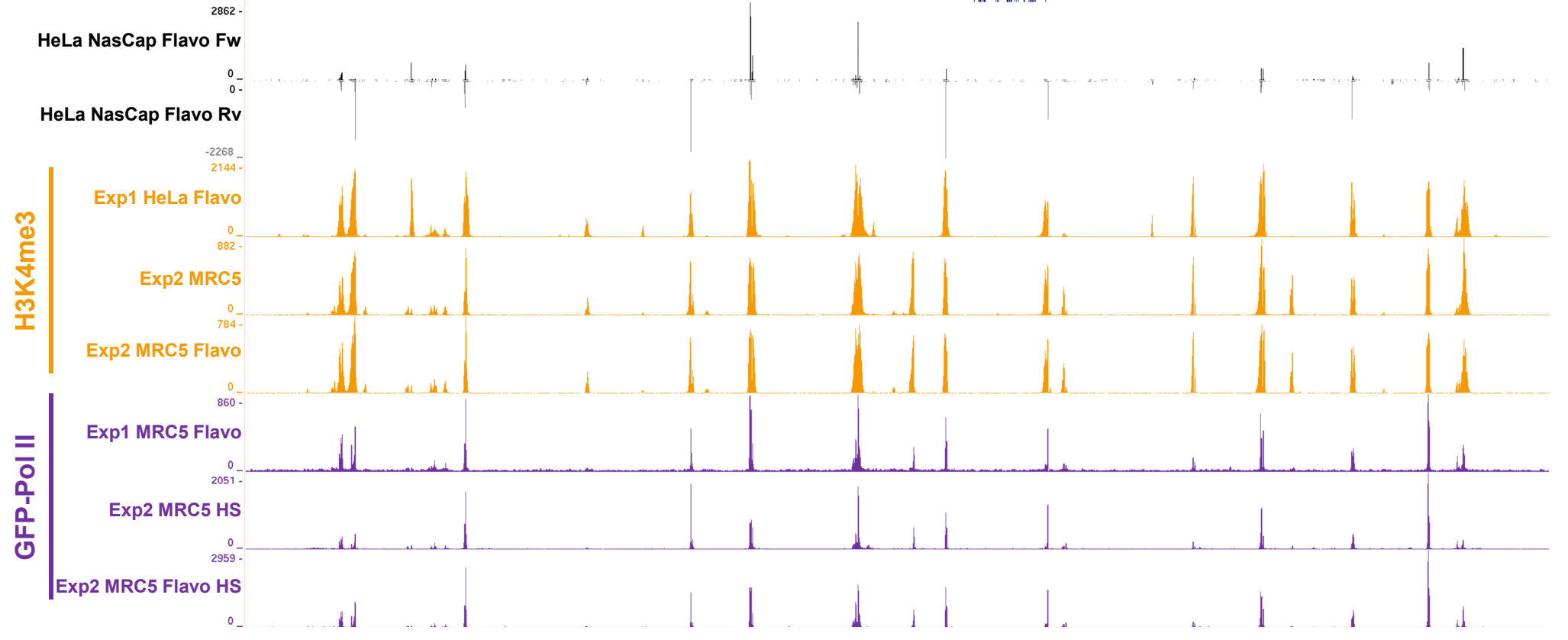

c
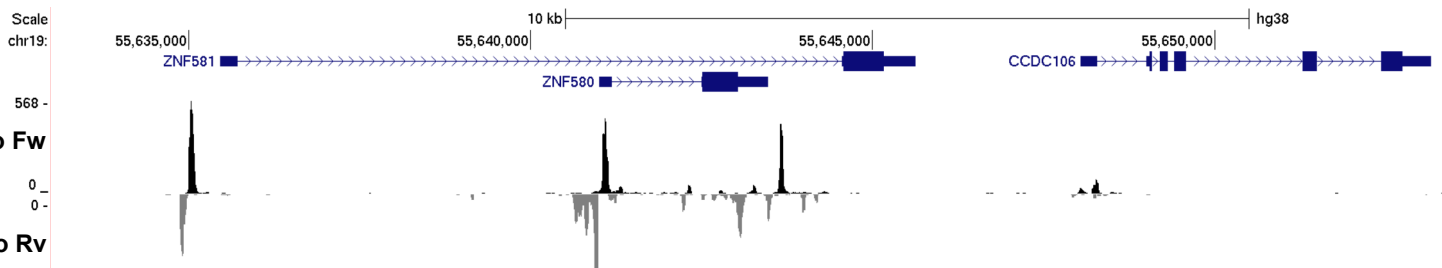

HeLa NasCap Flavo Fw

(2)

HeLa NasCap Flavo Rv
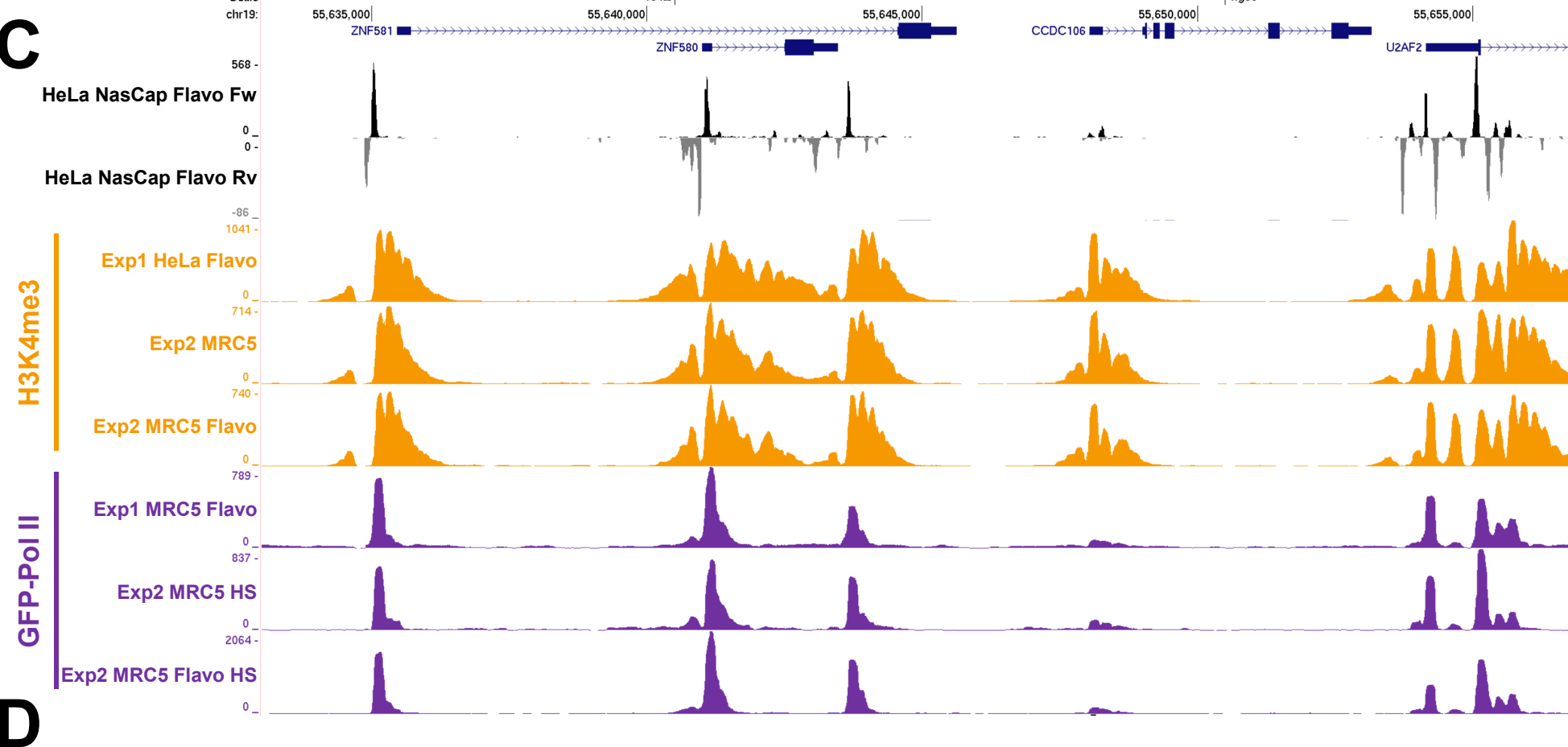

Exp1 HeLa Flav

vat -
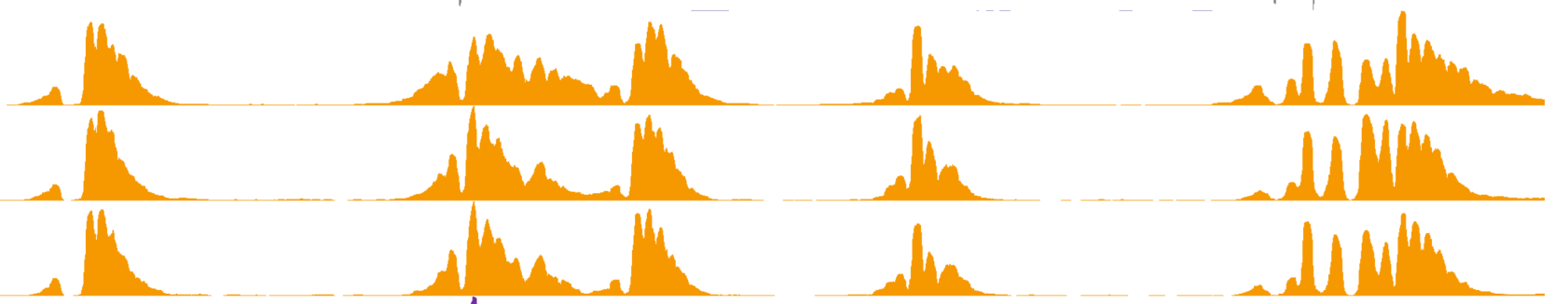

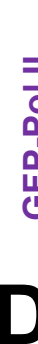

Exp1 MRC5 Flavo
037
Exp2 MRC5 HS



Exp2 MRC5 Flavo HS

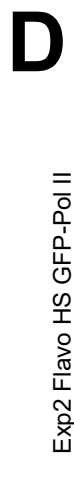


$\begin{array}{lllllll} & 50 \mathrm{~kb} \\ 41,880,000 & 41,890,000 & 41,900,000 & 41,910,000 \mid & 41,920,000 & 41,930,000 & 41,940,000\end{array}$ 59 -

$41,860,000-41,870,000$

$41,950,000 \mid \quad 41,960,000$

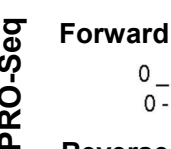

Reverse

을 $387^{-}$

鲧

ֻ̊ำ H3K4me3

Pol II

0
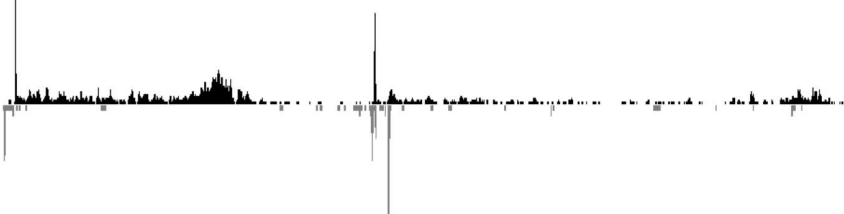

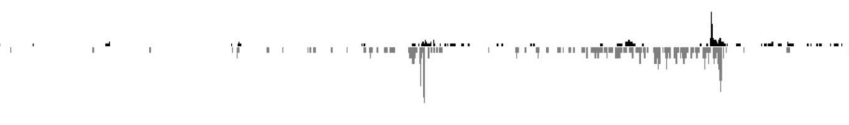

DMRTC2

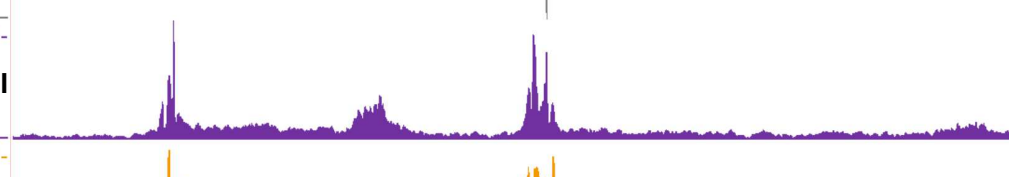

B

B scale $\mathrm{HCMV}$

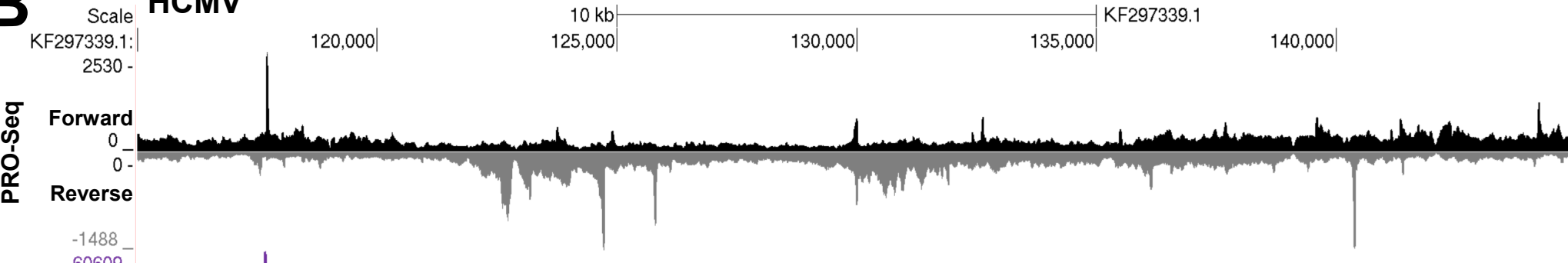

60609

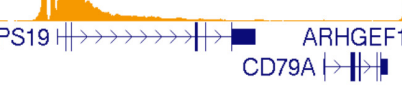

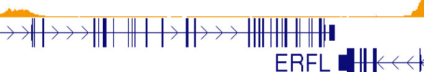
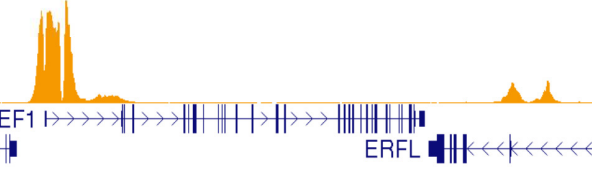

RABAC1 \|

C010616.1 K

ATP1A3 INH

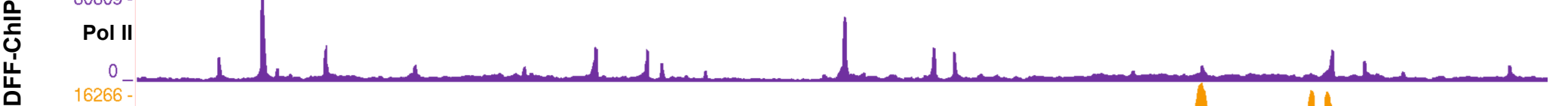

\% $\mathrm{H} 3 \mathrm{~K} 4 \mathrm{me3}$

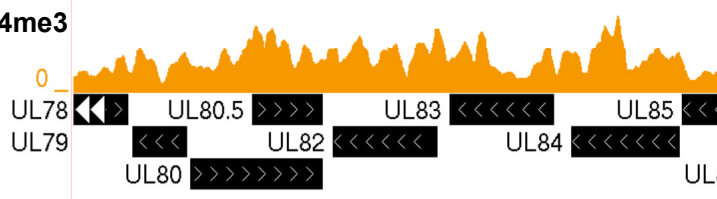

C

\section{Infected HFF}

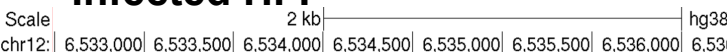

\begin{tabular}{|l|l|l|l|l|l|} 
&
\end{tabular}

orward

O̊

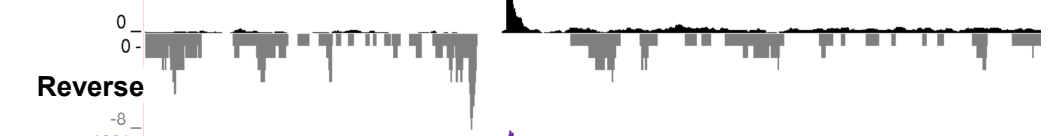

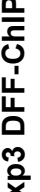

1331

Pol II

H3K4me3

岗

$\mathrm{E}$

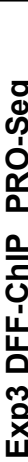

\section{HCMV}

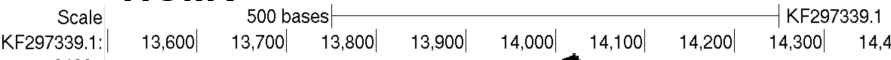

Forward

Forward

rse

Revers

$25285-$

Pol II

H3K4me3

UL94 $\gg>>$

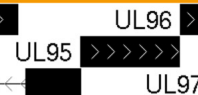

UL97 $\gg>>>>>>>$

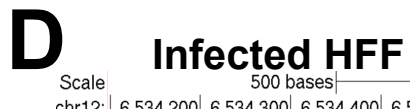

Scale 500 bases $\mid$ hg38

chr12:| 6,534,200| 6,534,300| 6,534,400| 6,534,500| 6,534,600| 6,534,700| 6,534,800| 6,534,900| 6,535,000|
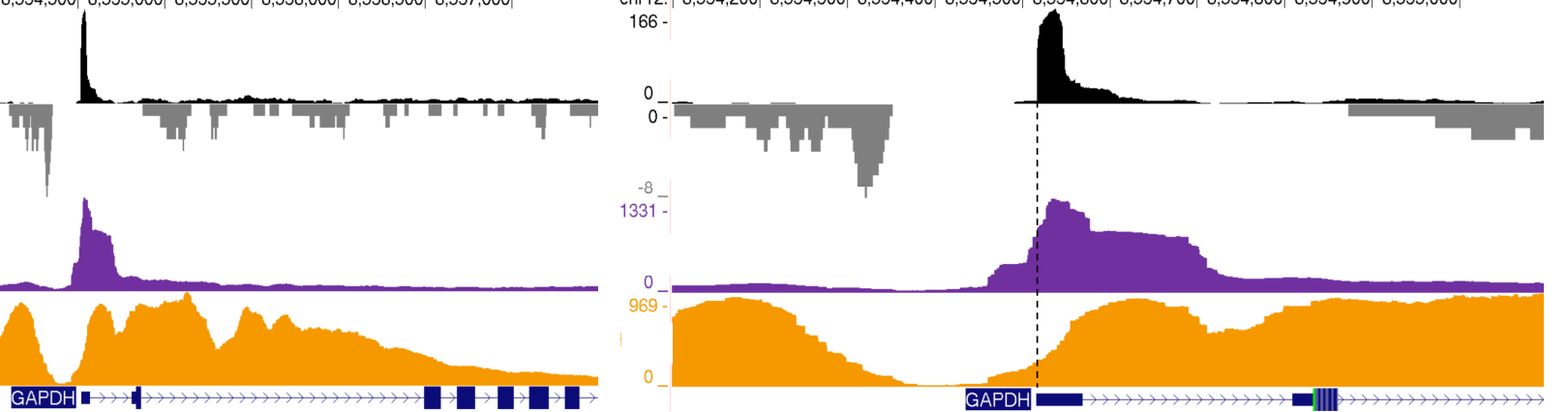

GAPDH

Figure 2 


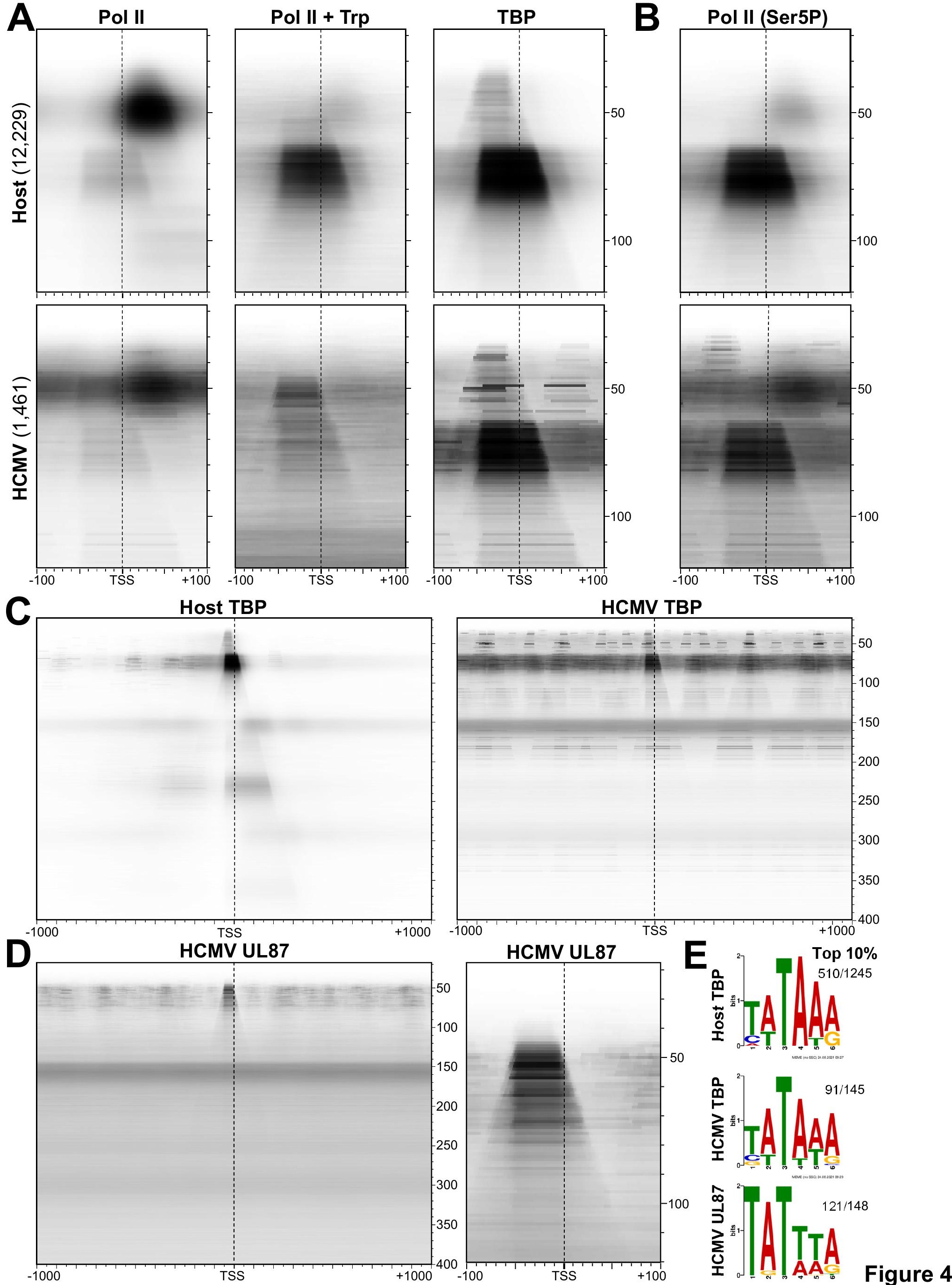


H3K4me3
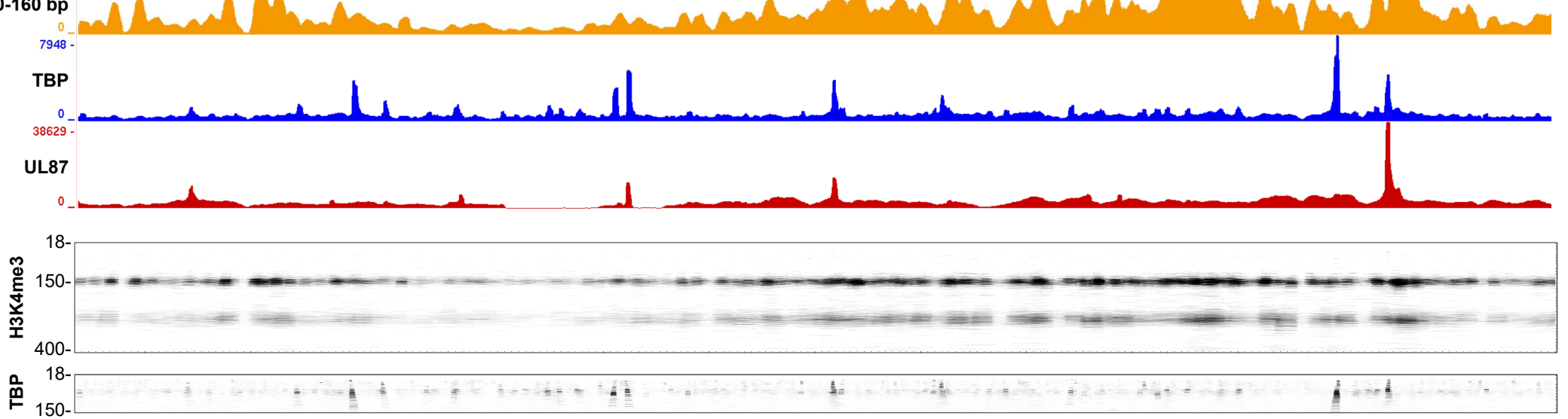
$\infty$

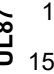

,

H3K4me3 positioning around TSSs

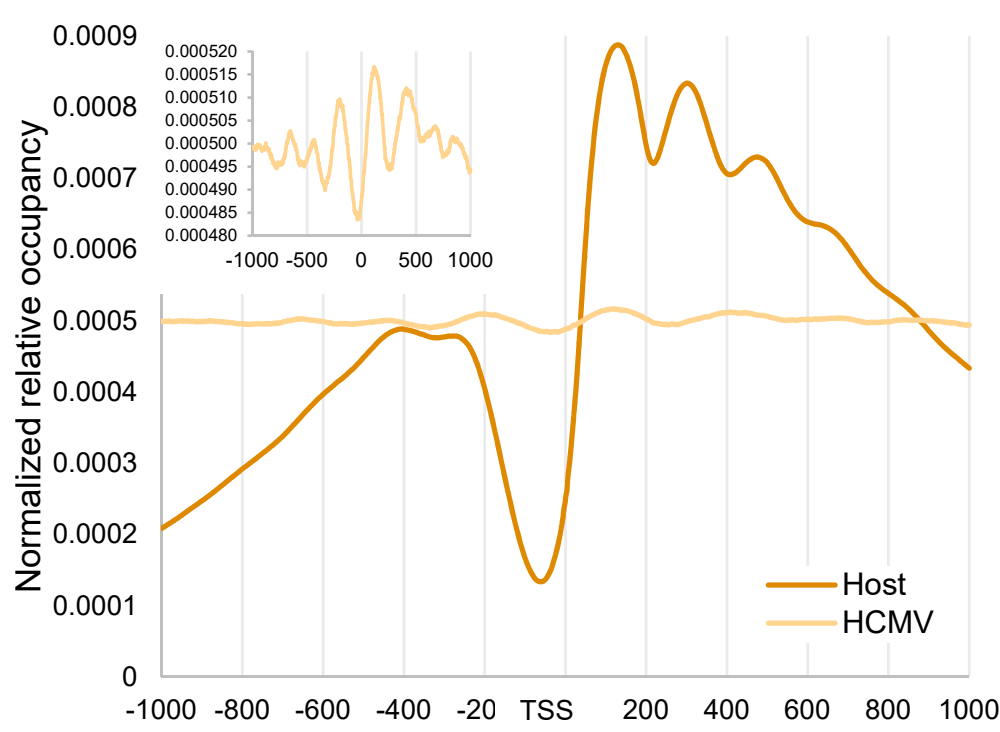

C

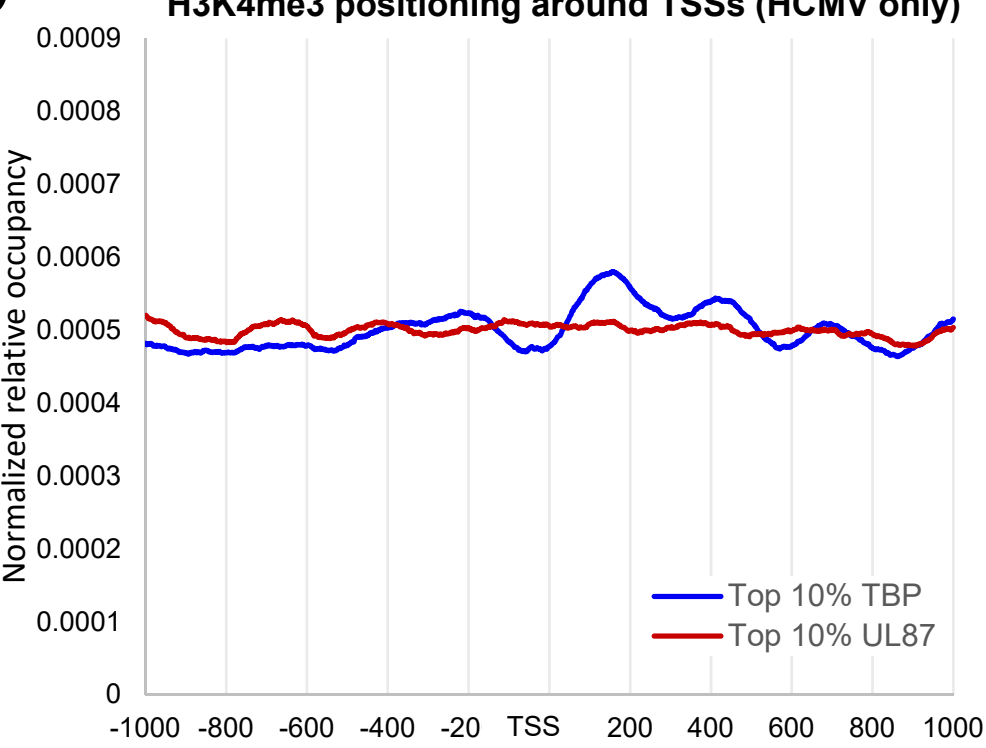

Figure 6 


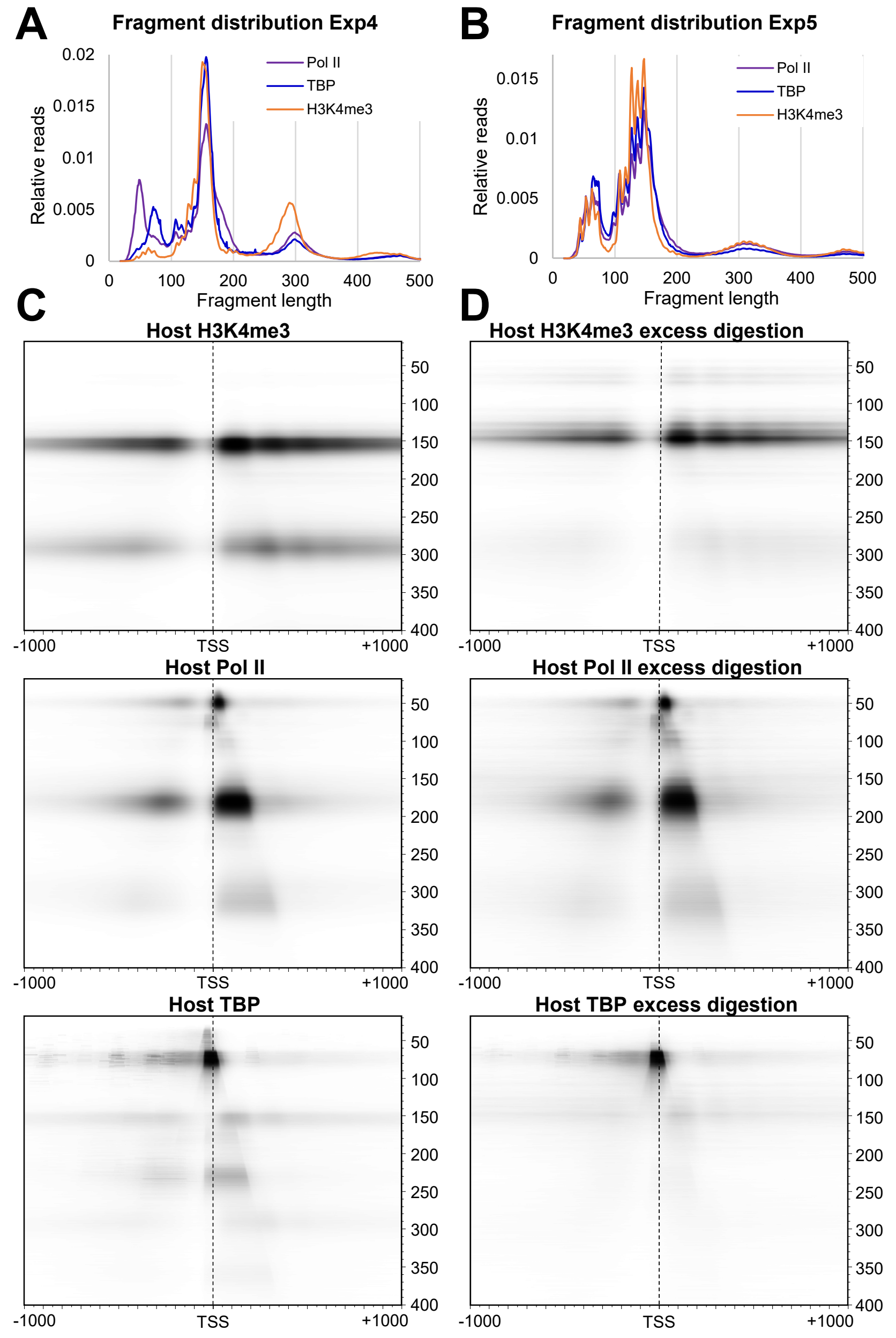


Normalized fragment length distribution

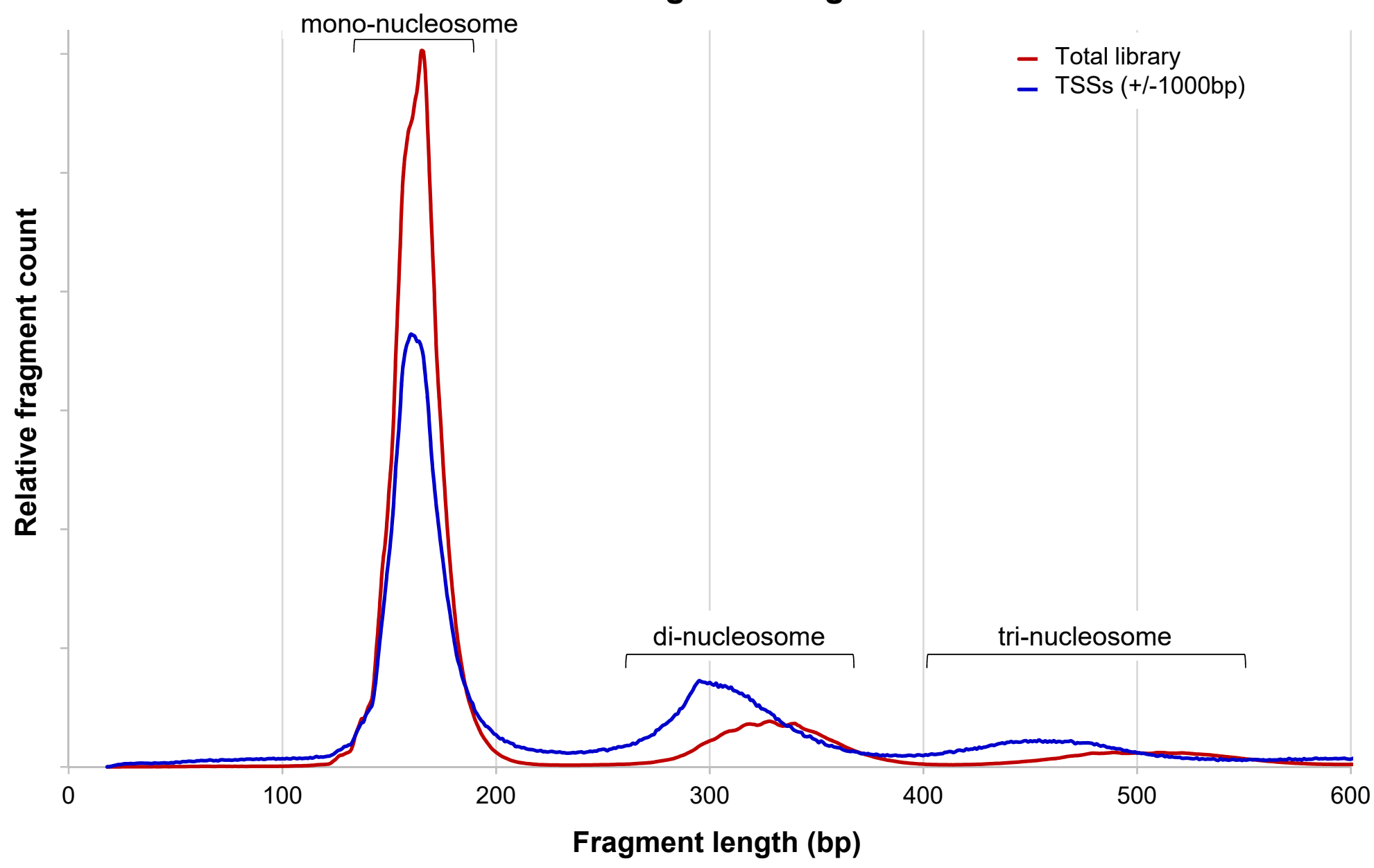

B

HeLa DFF-Seq

Base distribution at cut sites

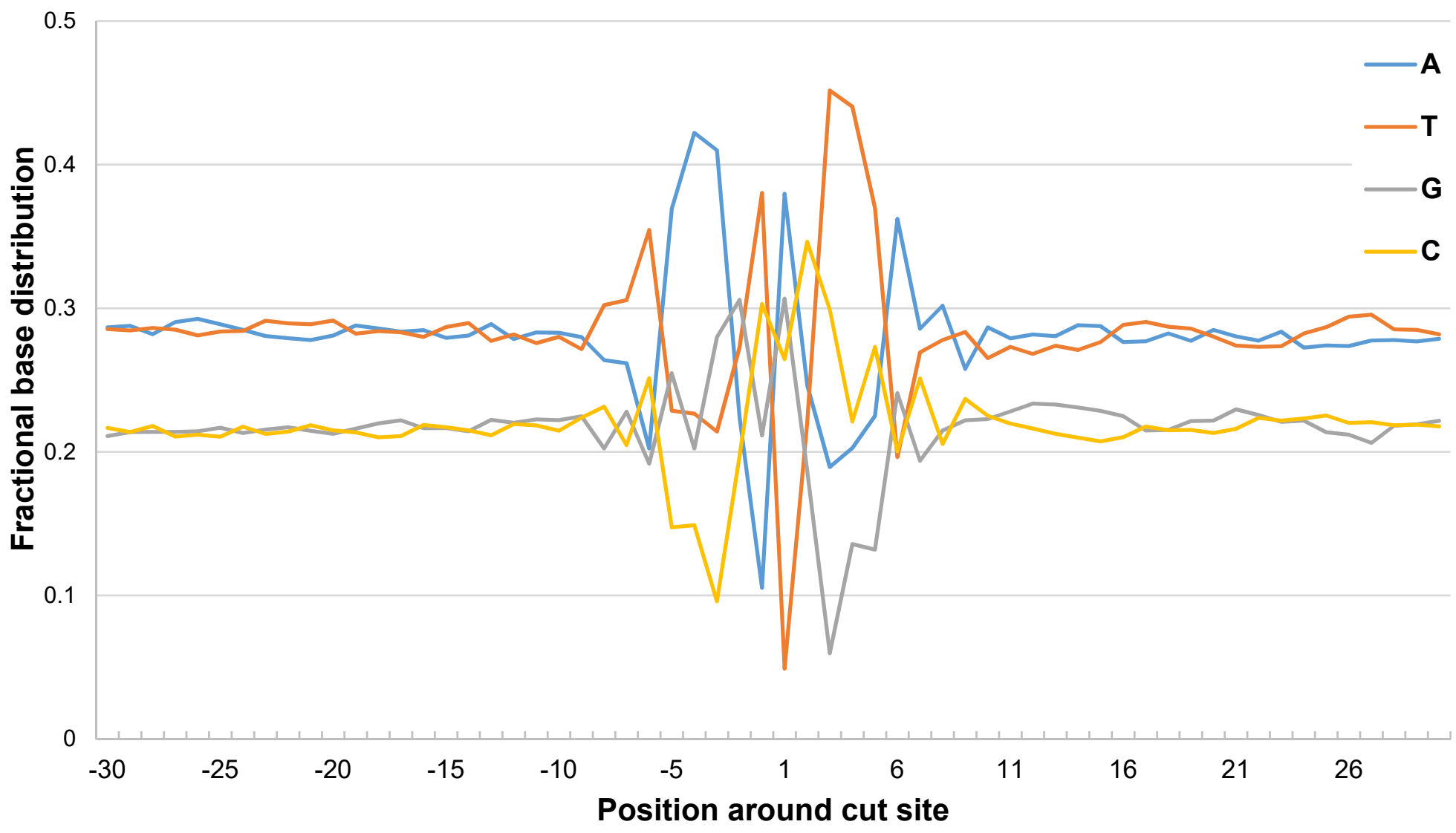

Figure S1 
A

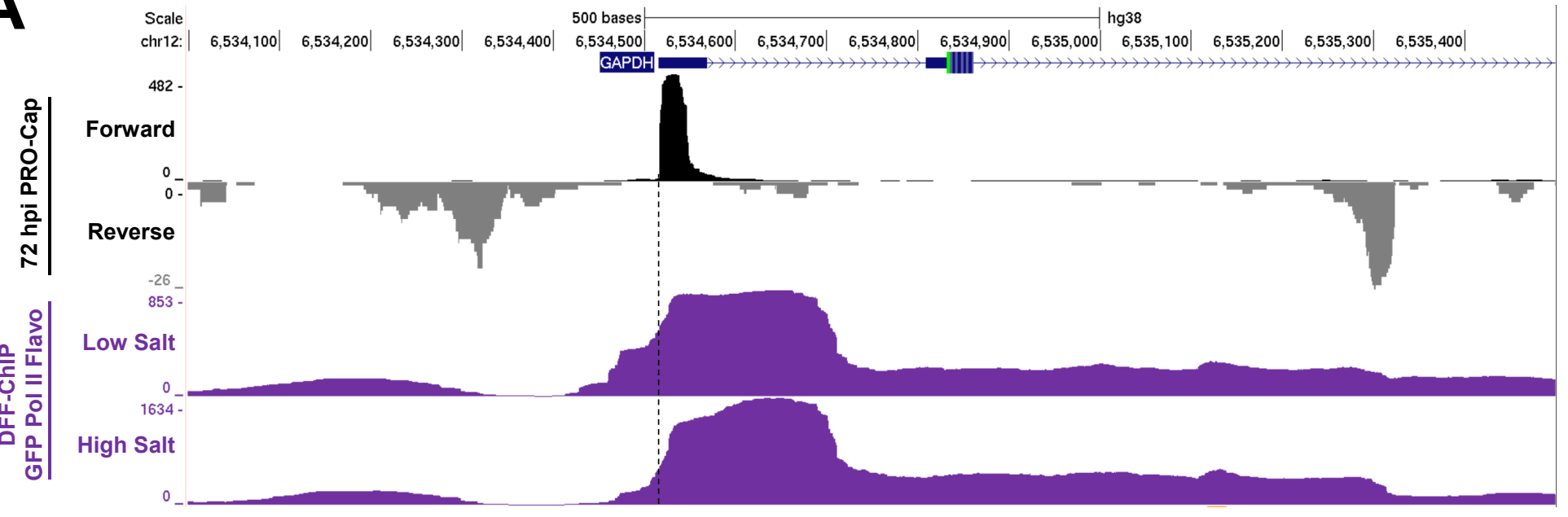

B

MRC5 GFP-Pol II Low Salt
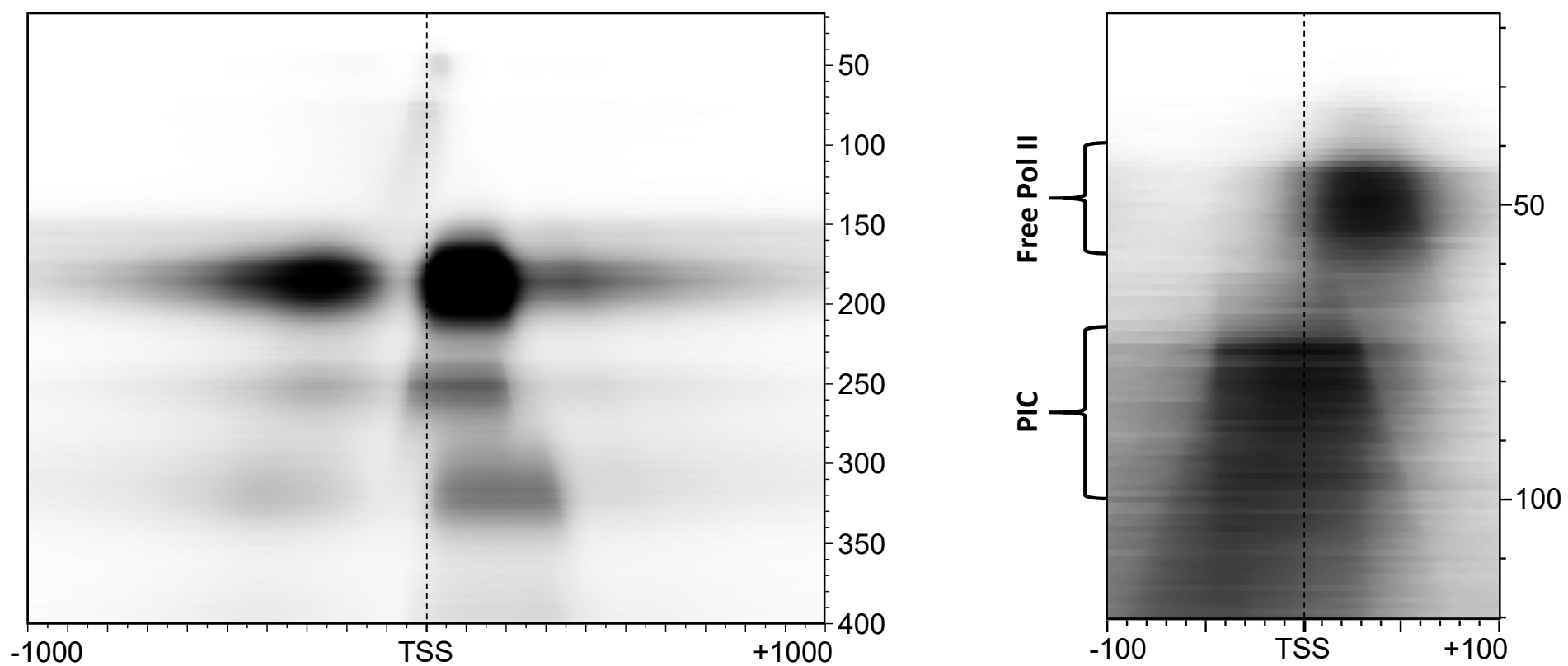

MRC5 GFP-Pol II High Salt
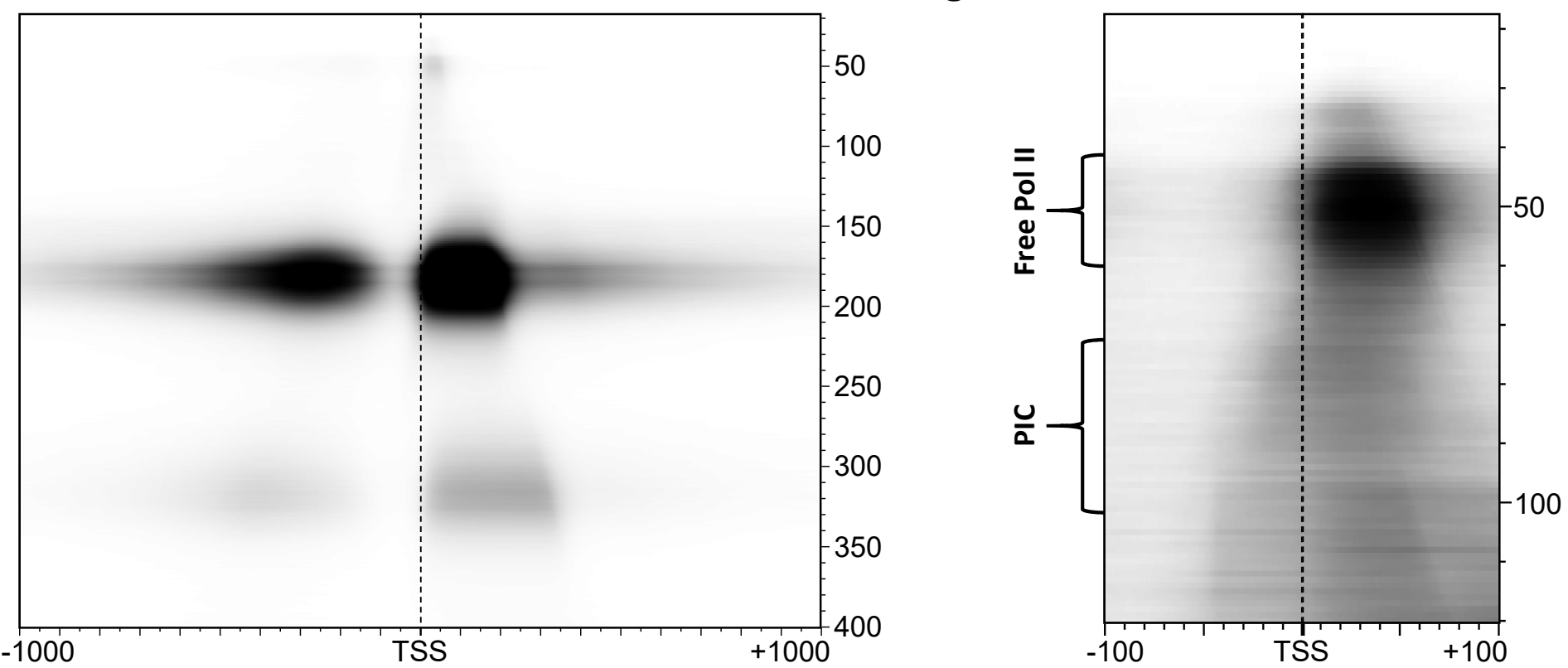

Figure S4 


\section{A Transcription levels at designated promoters}
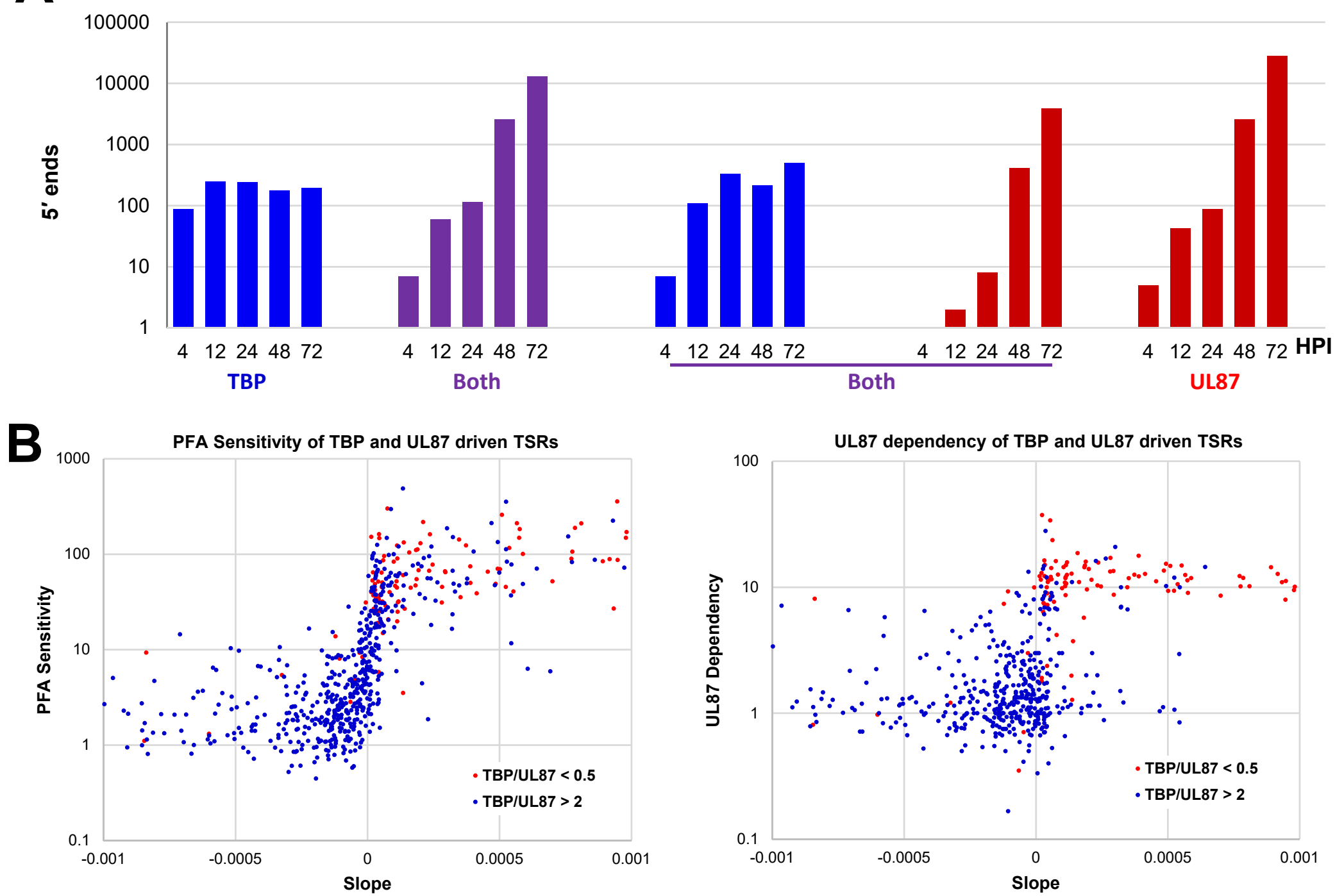

C

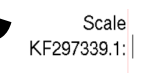

161 -

4 hpi Fw

221.-

12 hpi Fw

24 hpi Fw

$0-$
48 hpi Fw

48 hpi Fw

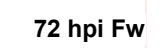

72 hpi $^{1782 .-}$

Flavo/PFA Fw
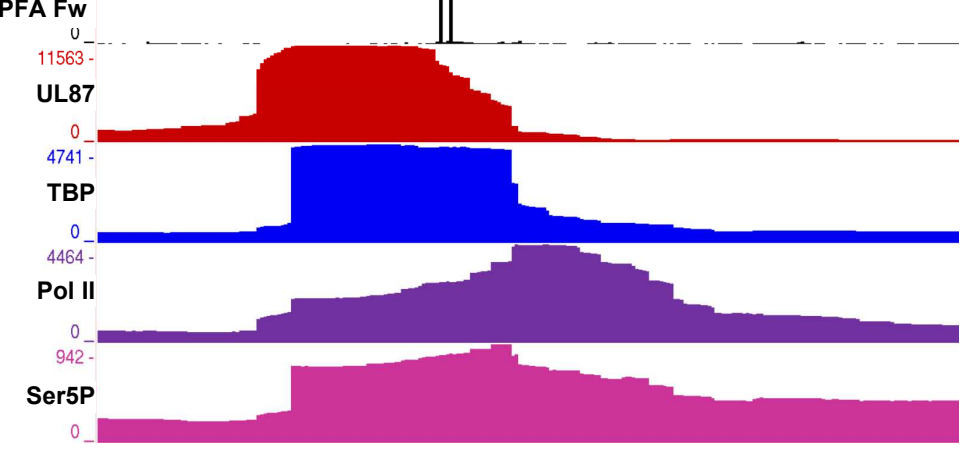

D
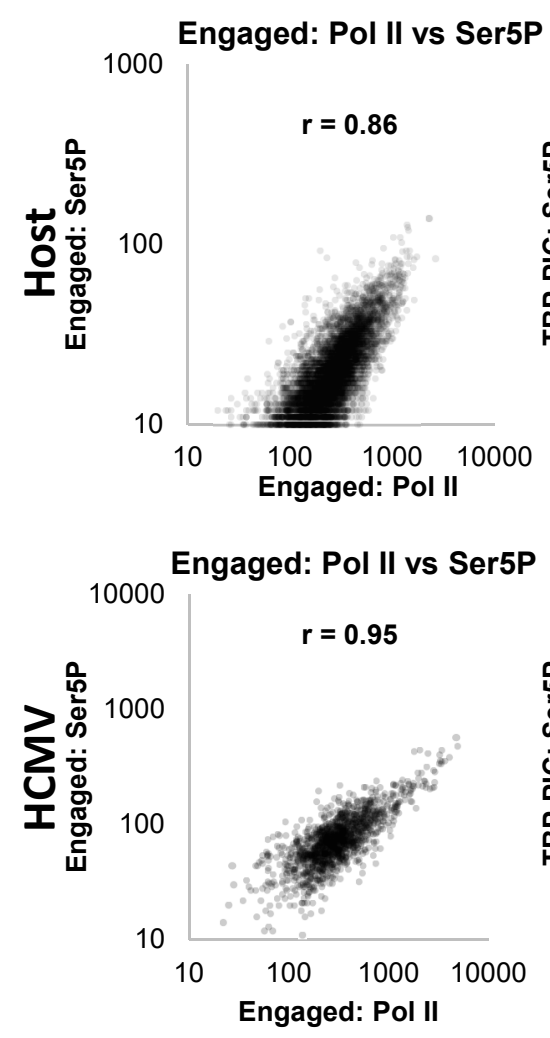

TBP PIC: TBP vs Ser5P

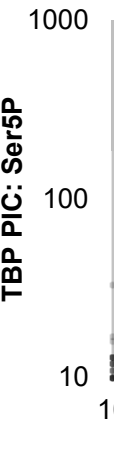

P 10000

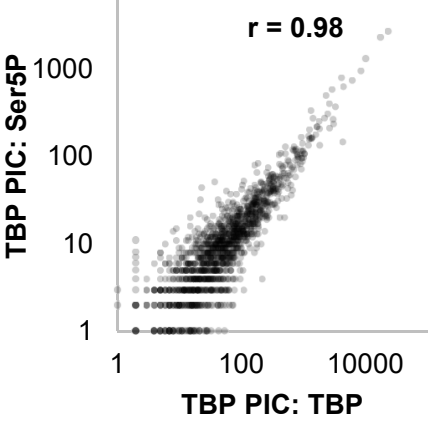

Figure S5 
$=$

TBP

UL87

H3K4me3

TBP

UL87

H3K4me3

TBP

UL87

H3K4me3

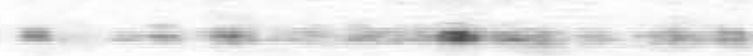

$+$

$+$

*

1

1

$1-21,000$

$20,000-$

41,000

TBP

UL87

H3K4me3

TBP

UL87

H3K4me3

TBP

UL87

H3K4me3

TBP

UL87

H3K4me3

TBP

UL87

H3K4me3

TBP

UL87

H3K4me3

TBP

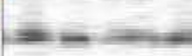

UL87

H3K4me3

TBP

UL87

H3K4me3

TBP

UL87 


\section{Supplementary Files}

This is a list of supplementary files associated with this preprint. Click to download.

- DataAnalysis.xIsx 